\title{
Performance Evaluation of Routing Metrics for Community Wireless Mesh Networks
}

\author{
by \\ Nan Liu \\ A thesis \\ submitted to the Victoria University of Wellington \\ in fulfilment of the \\ requirements for the degree of \\ Master of Engineering \\ in Network Engineering. \\ Victoria University of Wellington \\ 2012
}





\begin{abstract}
With the growth of different types of Internet traffic there is a compelling need to provide better quality of service, especially, over the increasing number of wireless networks. Expected Transmission Count (ETX) is a high throughput route selection metric that measures link loss ratios. ETX of a path reflects the total number of packet transmissions (including retransmission) required to successfully deliver a data packet along that path. Expected Transmission Time (ETT) is an improvement of ETX. ETT of a path is a measure of the transmission time needed to successfully deliver a packet along the path. ETT measures the loss ratio and the bandwidth of the link. Both, ETX and ETT, in comparison to hop count, provide better route selection for routing protocols widely used in Wireless Mesh Networks (WMNs). Using minimum hop count to find the shortest path has been shown to be inadequate for WMNs, as the selected routes often include the weakest links.

This thesis presents a performance evaluation comparing hop count, ETX and ETT when used with the Optimized Link State Routing version 2 (OLSRv2) protocol. This study is based on the wireless mesh topology of a suburban residential area in New Zealand, and analyses the performance of three common Internet traffic types in terms of throughput, end-to-end delay, jitter and packet loss ratio, and presents findings that are closer to the perspective of what an enduser experiences.

Also, a grid network of 121 nodes was used to analyze how the metrics choose paths, the performance changes (for different path lengths) and other conditions that affect the performance of the three metrics.
\end{abstract}




\section{Acknowledgments}

This research was performed in the Network Engineering Research Group of Faculty of Engineering and Computer Science in Victoria University of Wellington. The Network Engineering Research Group's interests span various networking domains such as wireless communications, networking protocols and energy harvesting. I started working on this thesis when I joined the group, and I am grateful to the members of the group for lending me support and improving my research.

I am most grateful to my supervisor Professor Winston Seah, who has helped me throughout the course of my Masters degree. He has guided me towards a realistic and interesting research topic, provided support and valuable suggestions. Winston has impressed faculty and students alike, both as an advisor and researcher. He has taught me how to improve my research skills, how to identify/address a problem and, how to write technical journals. We have learnt a lot from him during our numerous discussions.

Wei Li is a senior lecturer in the school. He has given valuable suggestions and shared his experiences in the research field as one friend would to another.

Mike Lin and Dong Xia, my lab mates, for extending helping hands throughout the course of my study. They helped me understand the fundamentals and improved my coding skills. They worked along with me when I encountered programming bugs.

Casimirraj Subramanian, for proof reading my thesis which improved 
the thesis quality.

Information and Communication Networks Laboratory of Niigata University release the OLSRv2 module for QualNet that includes the ETX metric. It has been very useful from the beginning of my simulation. I would also like to thank IMAI Hiroei from the Information and Communication Networks Laboratory. We corresponded through emails, and each contact helped me understand the OLSRv2 module a little better.

Ross Whitcher of VillageNets for providing the network topology maps which were useful for plotting node locations in our simulations.

I want to thank everyone in the Network Engineering Research Group for making it a stimulating place to work and learn. My lab mates deserve special thanks for being patient with my questions. 


\section{Contents}

\begin{tabular}{lll}
\hline & Introduction & $\mathbf{1}$
\end{tabular}

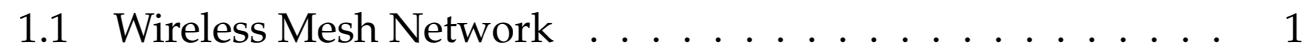

1.2 Routing Protocols and Metrics . . . . . . . . . . . . . 3

1.3 Internet Traffic . . . . . . . . . . . . . . . . . . 4

1.4 Routing Problems in WMNs . . . . . . . . . . . . . . . 4

1.5 Objectives ......................... 5

1.6 Contributions of this Work . . . . . . . . . . . 6

1.7 Thesis Structure $\ldots \ldots \ldots$. . . . . . . . . . . 7

$\begin{array}{llr}2 & \text { Related Work } & 9\end{array}$

2.1 Introduction . . . . . . . . . . . . . . . . . . 9

2.2 Routing Protocols . . . . . . . . . . . . . . . . 9

2.2 .1 Reactive Routing . . . . . . . . . . . . . . . . . . . . . . . . . . . . . .

2.2 .2 Proactive Routing . . . . . . . . . . . . . . . . . 11

2.3 Metric Characteristics . . . . . . . . . . . . . . . . . . 13

2.3 .1 Interference . . . . . . . . . . . . . . . 13

2.3 .2 Load Balancing . . . . . . . . . . . . . . . 15

2.3 .3 Locality of Information . . . . . . . . . . . . 15

2.3 .4 Isotonicity . . . . . . . . . . . . . . . 15

2.3 .5 Throughput . . . . . . . . . . . . . . . . . . . . . . . . . . . . . . . 16

2.3 .6 Route Stability . . . . . . . . . . . . . . 17

2.4 Routing Metrics . . . . . . . . . . . . . . . . . . 18

2.4 .1 Hop Count . . . . . . . . . . . . . . . 18 
$2.4 .2 \quad \mathrm{ETX} \ldots \ldots \ldots \ldots \ldots \ldots$

$2.4 .3 \quad$ ETT . . . . . . . . . . . . . . . 20

2.4 .4 WCETT . . . . . . . . . . . . . . . . . . . 21

2.4 .5 MIC . . . . . . . . . . . . . . . . 23

2.4 .6 Summary . . . . . . . . . . . . . . . . . 24

$\begin{array}{lll}3 & \text { Modification of OLSRv2 } & 27\end{array}$

3.1 Introduction . . . . . . . . . . . . . . . . . . . . . 27

3.2 Implementation of the ETX Metric . . . . . . . . . . . . 27

$3.2 .1 \quad$ Modification of the Hello message . . . . . . . . . 28

3.3 MPR algorithm modification . . . . . . . . . . . . . 28

3.4 Implementation of the ETT metric . . . . . . . . . . . 35

3.5 Summary . . . . . . . . . . . . . . . . . 35

4 A Grid Mesh Network Scenario 37

4.1 Introduction . . . . . . . . . . . . . . . . . 37

4.2 Simulation tool . . . . . . . . . . . . . . . . . . 37

4.3 A Grid Topology . . . . . . . . . . . . . . . . . . . . . . . 38

4.4 Simulation Environment . . . . . . . . . . . . . . . . . . . . . . 39

4.5 Traffic Initialization . . . . . . . . . . . . . . . . . . . . . . 40

4.6 Impact of Path Length $\ldots \ldots \ldots$

4.7 Impact of Flow Rate. . . . . . . . . . . . . . . . . . . 42

4.8 Impact of Node Density $\ldots \ldots \ldots \ldots$. . . . . . . . 47

4.9 Summary $\ldots \ldots \ldots \ldots \ldots \ldots \ldots$

5 Whitby Area Scenario 53

5.1 Introduction . . . . . . . . . . . . . . . . 53

5.2 Simulation Set up . . . . . . . . . . . . . . . 53

5.2 .1 Whitby Area Scenario . . . . . . . . . . . . . . . 53

5.2 .2 Simulation Environment . . . . . . . . . . . . . . . 55

5.3 Simulation Results . . . . . . . . . . . . . . . . . . . 56

$5.3 .1 \quad$ Voice over Internet Protocol (VoIP) Traffic . . . . . . . 56 
$5.3 .2 \quad$ File Transfer Protocol (FTP) Traffic . . . . . . . . . . . 61

5.3.3 Hypertext Transfer Protocol (HTTP) Traffic . . . . . . 63

5.3 .4 Summary . . . . . . . . . . . . 67

\begin{tabular}{llr}
\hline 6 & Conclusions & 69
\end{tabular}

\begin{tabular}{lll}
\hline 7 & Future Direction & 71
\end{tabular}

7.1 Improvement to the Simulation and Results Analysis . . . . 71

7.2 Real Network Experimention . . . . . . . . . . . . . . . 72

7.3 Multi-Radio and Multi-Channel Environment. . . . . . . . . 73 


\section{List of Figures}

$1.1 \quad$ An example of Wireless Mesh Network [4] . . . . . . . . . . 2

$2.1 \quad$ Traditional flooding and flooding using MPRs(blacks) . . . . 12

2.2 An example of intra-flow interference over a path . . . . . . 14

2.3 An example of inter-flow interference [23] . . . . . . . . . . . 14

2.4 An example of isotonicity [23] . . . . . . . . . . . . . . 16

$3.1 \quad$ An example of selecting flooding MPRs . . . . . . . . . . . . 29

3.2 Routing MPRs selection examples . . . . . . . . . . . . . . . . . . 30

3.3 Routing MPRs selection examples . . . . . . . . . . . . . . . 31

3.4 Routing MPRs selection examples [51] . . . . . . . . . . . . . 31

3.5 Routing MPRs selection examples [51] . . . . . . . . . . . . . 32

3.6 Selecting routing and flooding MPR sets . . . . . . . . . . . . 33

3.7 Selecting routing and flooding MPR sets [51] . . . . . . . . . 34

3.8 Selecting routing and flooding MPR sets [51] . . . . . . . . . 34

4.1 A 121 nodes grid network topology . . . . . . . . . . . . . . 38

4.2 First packet transmission time . . . . . . . . . . . . . . . 40

4.3 Average path length of CBR connections . . . . . . . . . . . 41

4.4 Average throughput with path length increases . . . . . . . . 42

4.5 Average throughput with various CBR flow rates . . . . . . 43

4.6 Average jitter with various CBR flow rates . . . . . . . . . . . 44

4.7 Packet loss ratio with various CBR flow rates . . . . . . . . . 45

4.8 Average end-to-end delay with various CBR flow rates . . . 46 
4.9 Average time of packets in queue . . . . . . . . . . . . . 46

4.10 Node density from 50 to 250 . . . . . . . . . . . . . . . 48

4.11 Throughput comparison with various node densities . . . . 49

4.12 End-to-end delay comparison with various node densities . 49

4.13 Jitter comparison with various node densities . . . . . . . . 50

4.14 Packet loss comparison with various node densities . . . . . 50

$5.1 \quad$ Whitby area network topology . . . . . . . . . . . 54

5.2 Average end-to-end delay . . . . . . . . . . . . . . . . . . . 57

5.3 Average jitter . . . . . . . . . . . . . . . 58

5.4 Average packet loss ratio . . . . . . . . . . . . . . . 59

5.5 Standard deviations of packet loss ratio . . . . . . . . . . 60

5.6 Number of control messages broadcast per node . . . . . . . 61

5.7 Throughput with number of FTP connections . . . . . . . . 62

5.8 Standard deviation of FTP connections' throughputs. . . . . 63

5.9 Throughput with number of HTTP connections . . . . . . . 64

5.10 Standard deviation of HTTP connections' throughputs . . . 65

5.11 Average page request time . . . . . . . . . . . . 66

5.12 Standard deviation of average page request time . . . . . . 66 


\section{List of Tables}

2.1 Metric Characteristics . . . . . . . . . . . . . . . . 26

4.1 Simulation Parameter . . . . . . . . . . . . . . . . . . . . . . . 39

4.2 OLSR and metrics Parameter . . . . . . . . . . . . . 40

5.1 Simulation Parameter . . . . . . . . . . . . . . . . . . 55 


\section{List of Abbreviations}

WMN Wireless Mesh Network

WANET Mobile Ad hoc Network

QoS Quality of Service

ETX Expected Transmission Count

ETT Expected Transmission Time

WCETT Weighted Cumulative Expected Transmission Time

MIC Interference and Channel switching cost

AODV Ad-hoc On-demand Distance Vector

DSR Dynamic Source Routing

DSDV Destination Sequence Distance Vector

OLSR Optimized Link State Routing

OLSRv2 Optimized Link State Routing version 2

LBAR Load-Balanced Ad hoc Routing

LQSR Link Quality Source Routing

MPR MultiPoint Relay 
MAC Medium Access Control

VolP Voice over Internet Protocol

CBR Constant Bit Rate

RTP Real-time Transport Protocol

FTP File Transfer Protocol

HTTP Hypertext Transfer Protocol

TCP Transmission Control Protocol

UDP User Datagram Protocol 


\section{Chapter 1}

\section{Introduction}

\subsection{Wireless Mesh Network}

Wireless Mesh Network (WMN) has been gaining considerable attention from industry and academia alike in recent years. As a result, researchers addressing various topics in WMN are prevalent in literature. Typically, WMNs are self-organized multi-hop networks comprising of stationary and mobile nodes. Nodes in a WMN consist of mesh routers and clients, both of which are capable of functioning as a router to extend the network reach or being end users. The connections between mesh nodes can be set up and maintained dynamically, thus making it a dynamic, self-coganized and self-healing network. WMNs can be classified into three categories based on the functionality of the comprised nodes: client backbone, infrastructure backbone and hybrid WMNs [1, 2].

In WMNs, mesh routers can be stationary or have minimal mobility and make up the backbone which facilitates network access for downstream mesh clients or routers. Also, by functioning as a gateway or bridge, Mesh routers can integrate existing wireless networks such as Wi$\mathrm{Fi}$, WiMax and sensor networks. Mesh clients can also function as routers to forward packets to other clients but they do not have gateway or bridge functionality and hence their software and hardware platforms are signif- 
icantly simpler than mesh routers [3]. Figure 1.1 shows an example of a Wireless Mesh Network.
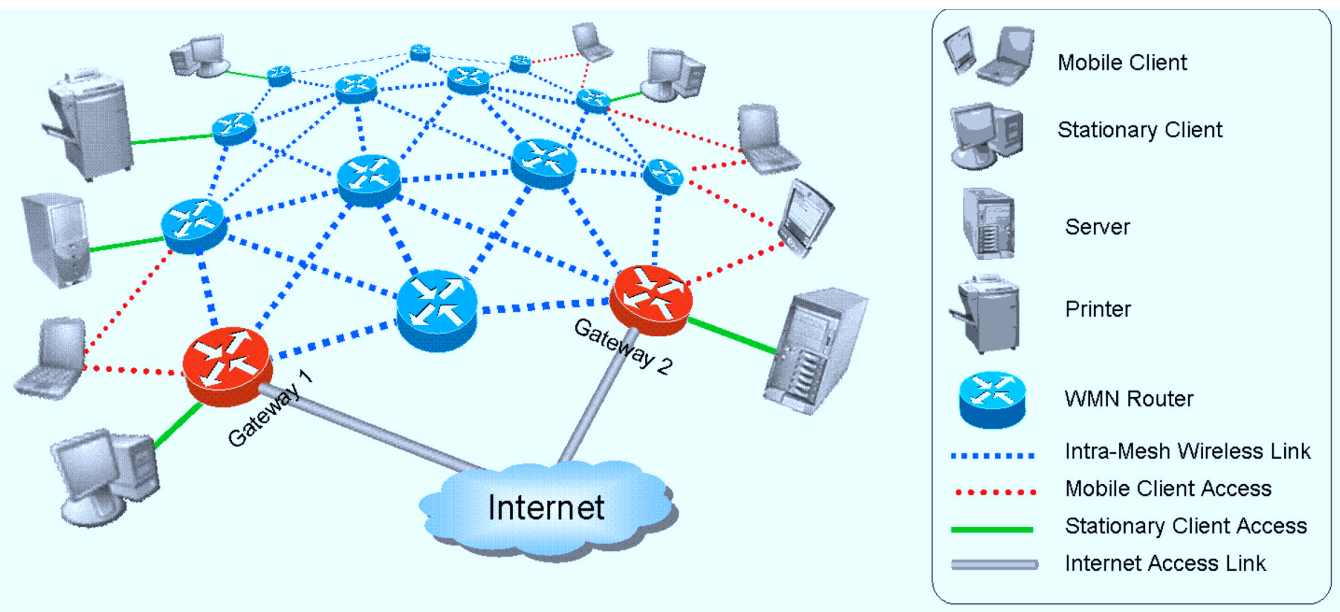

Figure 1.1: An example of Wireless Mesh Network [4]

Compared with traditional Wireless Local Area Networks (WLANs), the benefits of WMNs are as follows:

- High bandwidth: The physical characteristics of wireless links ascertain that shorter transmission ranges are more effective at utilizing bandwidth. This can be attributed to the increased interference and packet loss that longer transmission ranges evidently experience [3]. So, using multiple shorter hops to transmit data will offer higher bandwidth.

- Robustness: WMNs are more robust than one-hop networks. WMNs do not need a dedicated base station and hence rely less on the performance of a single node. In WMNs every node has multiple transmission paths so if one node or parts of the network fails, the operation of the entire network will not be affected as data can be transmitted along alternate paths.

- Flexibility: In traditional WLANs, clients have to share a common 
access point (AP). If they are required to access the Internet simultaneously it may lead to congestion and impact the performance of the network. Conversely, in WMNs, clients can access the Internet through multiple mesh routers; hence, the performance of the network will not be affected severely. Also, WMNs can utilize load balancing techniques through the use of multi-path routing. Different types of traffic can be assigned to disjoint paths according to their priority, thereby effectively reducing congestion.

\subsection{Routing Protocols and Metrics}

Routing protocols are an important entity in the design of a WMN as they hugely impact the performance of the network as a whole; hence, they have been a popular research topic for many years. WMNs' routing objectives are not very different from wired networks but, due to the wireless link characteristics, wired routing solutions cannot be used without modification. Many routing protocols designed for Mobile Ad hoc Networks (MANET) have been widely implemented in WMN and their performance studied [5, 6, 7]. Due to similar traits between MANETs and WMNs, adopted protocols such as Ad-hoc On-demand Distance Vector (AODV) [8] routing, Dynamic Source Routing (DSR) [9] and Optimized Link State Routing (OLSR) [10] work sufficiently well for WMNs. However, to further improve efficiency, designing an entirely new routing protocol for WMNs still remains an active research area.

The common purpose of routing protocols is to compute the best route for efficient data delivery. Weights are assigned to routes based on metrics to provide measurable values to judge how efficient a route will be. Different metrics will cause route selections to differ. Hence, to use or design an appropriate routing metric for a routing protocol, it is important to understand the characteristics of WMNs and identify what challenges will be faced. For example, a flow on one link may interfere with an adjacent 
flow because of the shared nature of the wireless medium. Therefore, to guarantee link quality, a good routing metric must take into account the metric characteristics to improve the performance of the routing protocol.

\subsection{Internet Traffic}

With the growth of the Internet, Voice over Internet Protocol (VoIP), bulk file transfer 1 which relies on File Transfer Protocol (FTP) [11] and web browsing traffic 2 which relies on Hypertext Transfer Protocol (HTTP) [12] constitute a signification portion of the Internet traffic. Users of applications that rely on these protocols expect at least the same quality level as in wired networks, and consequently, these protocols require higher Quality of Service (QoS) support over wireless links than what the shortest path routing can provide. While the shortest path routing has worked well for the wired Internet, when used in wireless networks, the selected routes often (if not always) include the weakest links. Consequently, any link quality fluctuation will result in recomputation of routes, incurring delays, packet loss and throughput degradation.

\subsection{Routing Problems in WMNs}

Most of current ad hoc routing protocols use hop count as their route selection metric. Hop count evaluates the suitability of a route/path purely based on the path length. It is simple and provides a high level of stability, and its isotonicity property allows it to find minimum weighted paths efficiently.

However, using hop count is not without limitations (discussed in Chapter 2), and it is insensitive to packet loss ratios, data rates, link capacity,

\footnotetext{
${ }^{1}$ In the following chapters, FTP traffic refers to bulk file transfer

${ }^{2}$ In the following chapters, HTTP traffic refers to web browsing traffic
} 
throughput, channel diversity, interference and various other routing requirements to assign weights. Much prior research [13, 14, 15, 16] represents the shortcomings of shortest path routing in multi-hop wireless networks. Therefore, a good routing metric should address the issues related to the key characteristics. For example, a route with good link quality and link capacity can provide better throughput and flow performance than a route with fewer hops (i.e. lower hop count) and a high loss ratio.

For different traffic types, users expect different quality of service over wireless links. VoIP traffic users experience a better quality of service when their traffic experiences less delay, jitter and low packet loss; FTP traffic users experience better service when the traffic stream achieves high throughput facilitating downloading/uploading data and HTTP traffic users experience better service when they are able to access websites faster. However, hop count by design does not satisfy these varying requirements, especially affecting performances and thus user experience as the traffic load gets higher. Different routing metrics designed for routing protocols can improve quality of service from the routing perspective and provide better user experience than hop count.

\subsection{Objectives}

This thesis will focuses on the the performance evaluation of three different metrics in a single radio environment by simulation. The aim of this thesis is to analyze the performance of three routing metrics with OLSRv2 protocol under different network conditions and different Internet traffic types. There are three goals in the thesis:

- Analyze and compare the performance of the hop count, ETX and ETT metrics under three Internet traffic types in a wireless mesh network based on a suburban residential area.

- Compare the path length of flows when using different metrics and 
analyze the performance with the flow rate changes in a grid topology network.

- Analyze the performance of three metric under realistic network topologies.

\subsection{Contributions of this Work}

The main contribution of this work is evaluating the hop count, ETX and ETT metrics with Optimized Link State Routing version 2 (OLSRv2) protocol using a realistic Community Wireless Mesh Network scenario by using Qualnet simulator. We used a part of the Porirua VillageNets Project deployment area, in the Whitby Area, as our simulation topology. We evaluated hop count, ETX and ETT metrics under three Internet traffic types by varying traffic loads. The topology is set up by using a part of Porirua VillageNets deployment topology to obtain more realistic results.

The ETX metric is a routing metric which takes into account the packet delivery ratio of each link in the route thus providing enhanced throughput. ETT metric is an extension of ETX that includes link capacity in addition to the packet delivery ratio. We used ETX and ETT metric instead of hop count in OLSRv2 protocol and compared results to distinguish performance differences brought about when using respective metrics. This enabled us to provide insightful analysis to further improve OLSRv2.

To evaluate what benefits routing metrics can bring to the common Internet traffic types, we evaluated hop count, ETX and ETT in terms of four characteristics of performance namely end-to-end delay, jitter, throughput and packet loss. We have identified these performance metrics as they have a significant impact on the common Internet traffic types in our study (Chapter 5). Also, the performance of the metrics under different network conditions are studied (Chapter 4).

A paper [26] based on a part of the thesis, entitled "Performance Eval- 
uation of Routing Metrics for Community Wireless Mesh Networks", has been accepted by the 7th International Conference on Intelligent Sensors, Sensor Networks and Information Processing (ISSNIP 2011), Adelaide, Australia, December 6-9, 2011. [ERA Rank B]

\subsection{Thesis Structure}

The structure of the thesis is as following:

- Chapter 2 provides background on routing protocols, metric characteristics and routing metrics in wireless networks. The most common routing metrics are compared and their advantages and disadvantages listed.

- Chapter 3 describes the modification of OLSRv2 protocol for implementing the ETX and ETT metrics and provides examples of how link metrics based on Multipoint Relays (MPR) algorithm work.

- Chapter 4 presents a grid mesh network scenario and realistic network scenarios to showcase the impact of path length, flow rate and node density to the three metrics.

- Chapter 5 evaluates how ETX and ETT improve the performance of three different traffic types using a part of Porirua VillageNets deployment topology.

- Chapter 6 and Chapter 7 are the conclusions and future work. 


\section{Chapter 2}

\section{Related Work}

\subsection{Introduction}

Routing protocols play an important role in Wireless Mesh Networks and has been an active research area for many years. All routing protocols are based on a specific routing metric. Ideally, these metrics need to consider certain routing benchmarks such as low level of interference, high throughput, link capacity, load balancing and channel diversity to guarantee better service. Every routing metric has its own advantages and disadvantages and, as such, may perform differently in varying topologies when used with different routing protocols. In this chapter, we will study and compare five widely researched routing metrics and two prominent routing protocols, and discuss their suitability for Wireless Mesh Networks.

\subsection{Routing Protocols}

Routing protocols take responsibility for route discovery, creation and maintenance of network topology. According to the time at which the routes are calculated, routing protocols are classified into two categories: reac- 
tive and proactive routing. Each of these routing models induces varying degrees of overhead on the network they are deployed in and directly affect throughput. Much prior research [17, 18, 19, 20, 21] has been done on WMNs. In this section, a brief introduction is provided for each of these routing models, following which a typical example is described in greater depth.

\subsubsection{Reactive Routing}

Reactive routing also called on-demand routing was originally proposed for ad hoc networks and includes protocols such as DSR, AODV and LoadBalanced Ad hoc Routing (LBAR) [22]. Reactive routing protocols only discover and establish a route when a source node has packets to send to a destination node. Reactive routing protocols usually use flooding to discover routes. In MANETs, high node mobility causes links to break and the network topology can change quite rapidly. Hence, compared with proactive routing protocols, on-demand route discovery limits control message overhead and provides higher network connectivity in MANET. However in WMNs, nodes are stationary and the frequency of link breakage is relatively lower, therefore network topology does not change frequently. In this scenario, deploying flooding-based routing discovery will only incur additional control message overheads and redundancy [23].

AODV is a typical reactive routing protocol. It uses a request-reply mechanism for route discovery [24]. When the source wants to send a packet to the destination, it will first check its routing table to see whether a route to the destination exists. If no route exists, it broadcasts a route request (RREQ) to its neighbors, who in turn forward the request to their neighbors until the destination or a node with a route to the destination is found. The RREQ message includes the hop count value, RREQ identifier (ID), source and destination IP addresses, and source and destination sequence numbers. The RREQ ID is used to identify a RREQ in order to 
avoid broadcast storms. Once a RREQ packet is received, a node will discard all further incoming RREQ packets of the same ID [25].

When a node forwards a RREQ packet, the hop count is increased by one. If there is more than one route, it will select the route with the least number of hops from source to destination. If the RREQ packet reaches the destination or an intermediate node with a valid route to the destination, the node will unicast a route reply (RREP) packet back to the source through the reverse path.

The route maintenance mechanism in AODV is triggered when a link breaks either due to node movement or transmission errors. The node detecting the break will send a route error (RERR) message to its active upstream neighbors to inform them of the link breakage and to update their route tables.

\subsubsection{Proactive Routing}

In a network deploying proactive routing protocols, such as OLSR and Link Quality Source Routing (LQSR) [26], each node maintains a routing table and periodically updates the status of its connectivity to every other node in the network. If the network topology changes or a link breaks, nodes will send update messages to the entire network in order to maintain up-to-date routing information.

The Optimized Link State Routing protocol (OLSR) is a proactive routing protocol based on the shortest path algorithm [27, 28, 29, 30]. Link state protocols use a flooding mechanism to exchange link state information. Nodes periodically broadcast a Link State Advertisement (LSA) message causing massive overheads, thus resulting in low network efficiency. In order to reduce overheads and redundancy during the routing creation and update process, OLSR uses a MultiPoint Relay (MPR) mechanism. In this mechanism, each node will select the smallest MPR set from its one hop neighbors with symmetric links, which can effectively provide connectiv- 
ity to all its two hop neighbors. Only the nodes selected as MPRs assume the responsibility of forwarding control messages to the entire network. This mechanism reduces the overhead caused by control messages and improves flooding efficiency, thereby decreasing retransmissions. Figure 2.1 shows the difference between traditional flooding a packet and flooding a packet using MPRs.
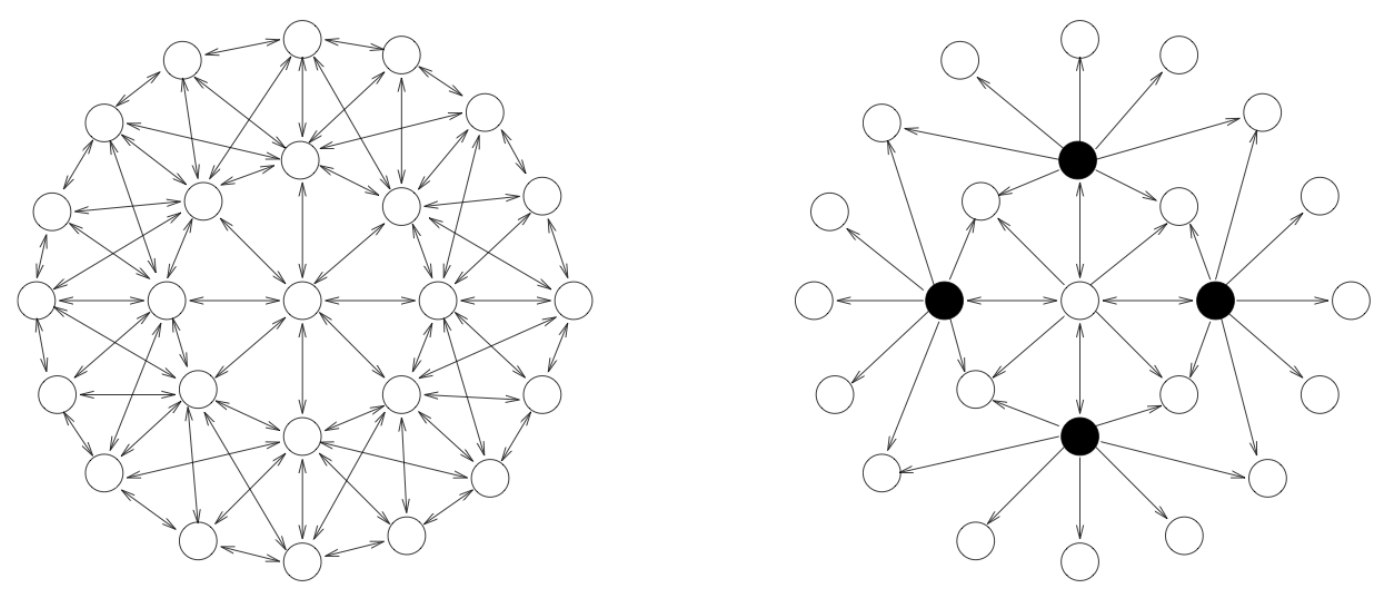

Figure 2.1: Traditional flooding and flooding using MPRs(blacks)

OLSR mainly uses two basic types of control messages: Hello message and Topology Control (TC) message. The Hello message serves to discover link information, 1-hop and 2-hop neighbors. It is also involved in the MPR selection process. These Hello messages are broadcast periodically, thereby enabling nodes to keep track of the immediate changes in their local neighborhood. TC messages are used to propagate topology information into the entire network, so that every node can use this information to calculate the shortest path to a desired destination. TC messages are broadcast periodically, but only nodes selected as MPRs can generate a TC message keeping overhead to a minimum. Every node in its 1-hop neighborhood selects the smallest set of MPRs to cover all its 2-hop neighbors. Thereafter, MPRs will announce to the network their reachability to the nodes that have selected them as an MPR. 
OLSRv2, proposed in [31], is an updated version that retains the same basic functions and algorithms as OLSR, while additionally providing more simplified messages and a flexible signaling framework.

\subsection{Metric Characteristics}

An effective routing metric has to capture the quality of network links in order to compute the best path. Each routing metric has its own advantages and disadvantages in meeting these criterion. They also need to ensure the stability of the network and allow for the computation of the minimum weight path. In this section, some important criteria are described.

\subsubsection{Interference}

Interference is a serious issue in wireless networks due to the shared nature of the wireless medium resulting in different flows competing for their share of the bandwidth. Interference in wireless networks usually is divided into two types: intra-flow interference and inter-flow interference

\section{Intra-flow Interference}

Intra-flow interference occurs if more than one link on the same path within a node's radio range uses the same channel [32]. Intra-flow interference reduces throughput and increases delay as the hop count increase. Hence an ideal solution is to increase channel diversity, i.e. selecting non-overlapping channels for the links on the same path within a node's radio range. A good routing metric must hence consider channel diversity.

For example, as shown in Figure 2.2, links $A \rightarrow B$ and $B \rightarrow C$ use the same channel, hence there is intra-flow interference between $A$ and B. However, 
in the case of link $\mathrm{A} \rightarrow \mathrm{D}$ and link $\mathrm{D} \rightarrow \mathrm{C}$ using different channel, there is no intra-flow interference.
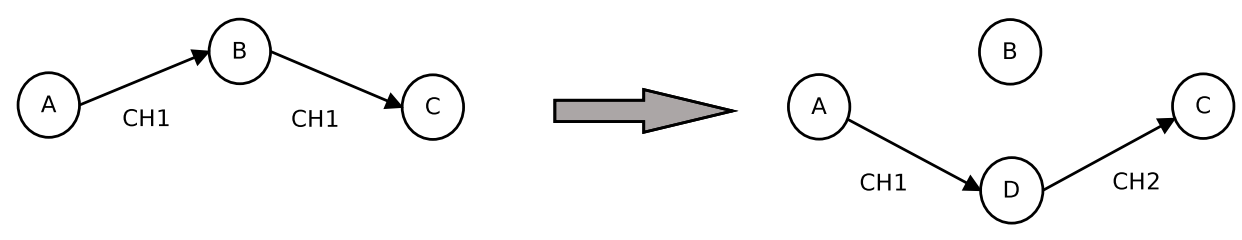

Figure 2.2: An example of intra-flow interference over a path

\section{Inter-flow Interference}

Inter-flow interference occurs when different flows operate on the same channel within radio range of each other, thereby competing for the medium. This may lead to bandwidth starvation, as affected nodes might sense the channel to be busy. Occurrence of inter-flow interference is hard to predict and hence effectively control, as it involves multiple flows over different paths.
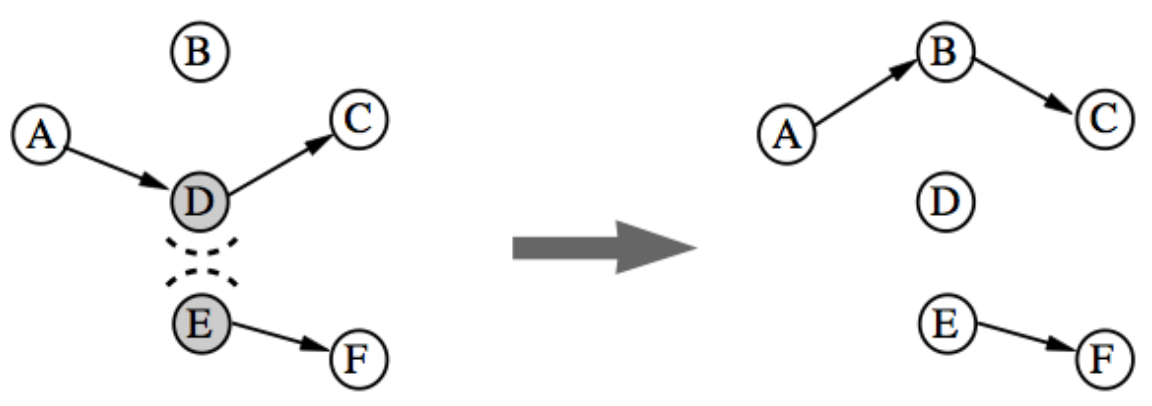

Figure 2.3: An example of inter-flow interference [23]

Figure 2.3 shows paths $\mathrm{A} \rightarrow \mathrm{D} \rightarrow \mathrm{C}$ and $\mathrm{E} \rightarrow \mathrm{F}$ using the same channel, and as a result, they experience inter-flow interference with each other. If the path $\mathrm{A} \rightarrow \mathrm{D} \rightarrow \mathrm{C}$ changes to $\mathrm{A} \rightarrow \mathrm{B} \rightarrow \mathrm{C}$, they will be less inter-flow interference. 


\subsubsection{Load Balancing}

A metric should provide fair usage of the network resources. Normally, a metric will maximize the throughput along an individual path, which has already been established, and does not take into account the entire network performance. The reason for this is routing metrics consider the link capacity when selecting a route, and do not consider the prevailing link load. This leads to unfair usage of bandwidth and wastage of network resources [33].

Instead, routing metrics should use real-time information on link capacity gathered from neighboring nodes, to make a choice so as to avoid the highly loaded paths and thus minimize the impact on neighboring flows. This can be achieved by finding routes that are underutilized [34].

\subsubsection{Locality of Information}

For calculating more optimal routes, metrics require global information such as packet loss ratio, delay and channel information that depicts the current state of the links in the network. For example, when using ETT as the routing metric, every node needs to utilize the ETT value of other links while calculating paths. The gathering of such global information cause two issues: one is the network is flooded with control messages which are used to propagate information to every node on the network. These excessively frequent and abundant control messages increase network overhead; another is when establishing routes, delay will increase as processing overhead increases due to increased control messages [32].

\subsubsection{Isotonicity}

Isotonicity [35, 36] of a routing metric means that the orders of the weight of two paths do not change when appended or prefixed by a common third path. If a routing metric does not account for isotonic property, links 
might not be stable when routing algorithms use it to calculate minimum weight paths. For example, if there is a common additional path that is added to two different paths, the minimum weight of the paths might vary. Hence, it is important that a routing metric takes into account the isotonic property.

Figure 2.4 gives an example of isotonicity. $\mathrm{W}(\mathrm{x})$ refers to the weight of a path $\mathrm{x}$. There are two connections $a$ and $b$. The definition of isotonicity is if $W(a) \leq W(b)$, then $W(a \oplus c) \leq W(b \oplus c)$ and $W\left(c^{\prime} \oplus a\right) \leq W\left(c^{\prime} \oplus b\right)$.

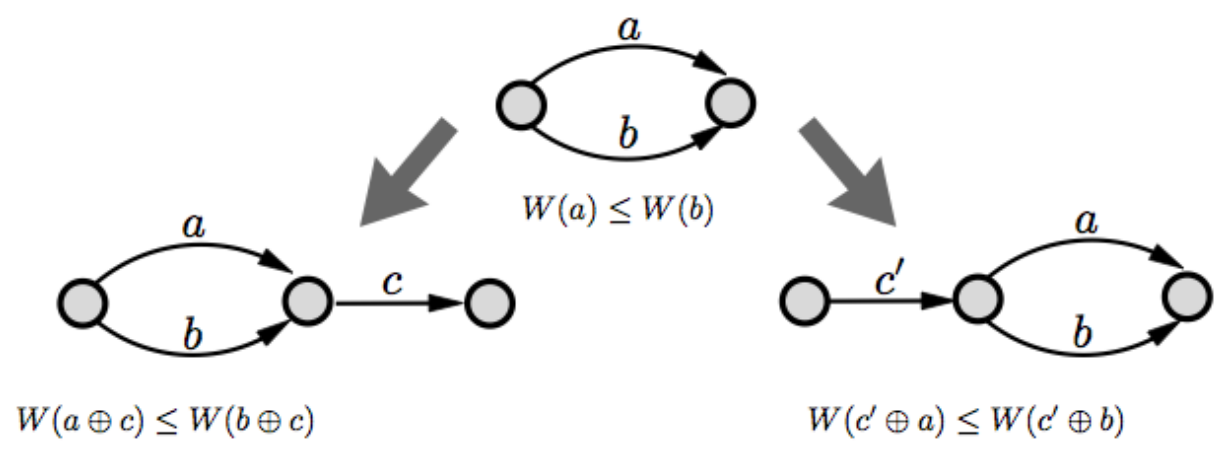

Figure 2.4: An example of isotonicity [23]

\subsubsection{Throughput}

When selecting routes, routing metrics should consider throughput. This is because a high throughput path can guarantee a faster transmission rate and nodes can make better use of the wireless medium. High throughput can also lessen delay and improve performance. However, throughput can be affected by many factors. For example, with the increase of path length, the probability of loss due to faulty links increases and might result in throughput of the flow being reduced. Hence, an effective routing metric must ensure a good throughput along a path. 


\subsubsection{Route Stability}

The performance of a network is easily affected by unstable path weights. Frequent changes can trigger protocols to generate copious update messages and cause the network to incur high overheads.

The stability of path weights, which can be captured by the routing metrics, can be divided into two types: load-sensitive and topology-dependent [23]. In load-sensitive metrics, a weight is assigned to a route based on the load of the traffic along the route, such as Interface Switching Cost [37] and Number of Congested Node [38] . If the weight of a route is assigned using load-sensitive metrics, it may change frequently as traffic flow fluctuates. By contrast, in topology-dependent metrics, a weight is assigned to a route based on the topology elements, which include link capacity and hop count. Since the topology of mesh networks do not change frequently, topology-dependent metrics might result in more stable routes.

Load-sensitive and topology-dependent metrics deal with path weight tolerance differently when deployed with different routing models. For reactive protocols, the route is discovered/established only when a flow is initiated. The weights of these established routes are not recalculated during the course of the transmission, except for when route errors occur and rerouting is required. Therefore, changes in load-sensitive metrics of reactive protocols do not induce flows to change their routes and negatively affect the stability of the network. Hence, load-sensitive metrics are more suitable for reactive routing protocols.

On the other hand, proactive routing protocols update routes periodically. A change in routing metrics may lead to route updates during the lifetime of the flow. This will also happen in cases of reactive routing protocols. Load-sensitive routing metrics have a high risk of instability, since large and irregular traffic variations along the route will result in the path weights changing frequently. For this reason, topology-dependent routing metrics are more appropriate for mesh networks, as they can be used for both proactive and reactive routing protocols. 


\subsection{Routing Metrics}

A good routing metric should address the issues related to the key characteristics (discussed in 2.3) such as interference, isotonicity and throughput. In this section, we analyze five routing metrics that have been developed for WMNs to understand how they satisfy the requirements for routing. The routing metrics described below are all, topology-dependent and, each one provides an improvement over those proposed earlier.

\subsubsection{Hop Count}

Hop count is the fundamental and most common routing metric in conventional networks, including WMNs, and allows us to determine how many hops away the destination is from the source. Many routing protocols such as DSR, AODV, OLSR and Destination Sequence Distance Vector (DSDV) [39] use hop count as their base metric.

Hop count evaluates the suitability of a route based purely on the path length. It is simple and provides a high level of stability, and its isotonicity property allows it to find minimum weighted paths efficiently.

However, hop count is not without limitations and these include ignoring packet loss ratios, data rates, link capacity, throughput, channel diversity, interference and various other routing requirements to assign weights. For example, a route with good link quality and link capacity can provide better throughput and flow performance than a route with fewer hops (i.e. lower hop count) and a high loss ratio.

\subsubsection{ETX}

Expected Transmission Count (ETX) [40] is a metric that measures link quality by estimating the number of transmissions and retransmissions needed to send a data packet over a link. To get the ETX value, every node broadcasts a probe packet periodically to neighboring nodes. The weight 
of the total path is the summation of each link's ETX value along the length of the path. The formula to calculate ETX is given below:

$$
E T X=\frac{1}{d_{f} \times d_{r}}
$$

Where, the forward delivery ratio, $d_{f}$, denotes the probability that a packet will be successfully delivered in the forward direction, and the reverse delivery ratio $d_{r}$ denotes the probability of receiving the corresponding acknowledgement packet.

Therefore, ETX involves the delivery ratio and the number of transmissions in both directions over a link. Since the two probabilities are independent, $d_{f} \times d_{r}$ can be understood as the expected probability of a successful transmission, which includes acknowledgement. $d_{f} \times d_{r}$ is also equal to $\left(1-P_{f}\right) \times\left(1-P_{r}\right)$, where $P_{f}$ and $P_{r}$ are the forward and reverse packet loss ratios.

Link ETX uses probe measurement for the calculation of delivery ratios. Each node broadcasts small link probes (134 bytes) once every second. As broadcast packets are neither retransmitted nor acknowledged at the IEEE 802.11 Medium Access Control (MAC) layer, a node remembers the probes it receives from its neighbors during a sliding window of $\mathrm{du}$ ration of $\omega$ seconds (usually $\omega=10$ ). Nodes also send the record during the same sliding time window to their neighbors, so that at any given time a node can calculate the delivery ratio in both directions. To avoid the possible synchronization of periodically broadcast probe packets, which could lead to large-scale collision, a random jitter is used for every probe packet. The jitter value is usually $\pm 0.1 \omega[41]$.

Since the value of the ETX metric is based on the delivery ratio, it directly affects throughput and packet loss ratio in both directions of a given link. This will imply that a path with low ETX value has low congestion, low packet loss ratio, and hence high throughput. ETX uses broadcast instead of unicast, thereby reducing probing overheads. ETX indirectly handles inter-flow interference, because a high level inter-flow interfer- 
ence causes high packet loss ratio. ETX is also isotonic and therefore it is easy for routing metric to calculate the minimum weight path.

ETX performs better than hop count in single-radio and single-channel WMNs. However, for multi-channel WMNs, ETX does not have the ability to deal with intra-flow interference. It may select a lower transmission rate increasing time to transmit data but neighboring nodes will have to back off of their transmission [32]. ETX also does not consider link load. When a route passes through heavily loaded nodes, this will cause unbalanced resource usage.

The main disadvantages of ETX lie in the way it broadcasts small probe packets to detect data delivery ratio, and that probe packets are sent at a lower data rate. Data delivery estimates may not reflect the real packet loss ratio, because actual packets are usually larger and sent at higher data rates. Additionally, ETX does not take link data rates into account. Identical packet loss ratio may be associated with different data rates and link delays. For this reason, ETX is more suitable for single-rate networks.

\subsubsection{ETT}

The Expected Transmission Time (ETT) metric, proposed in [42] is an improvement of ETX, and it addresses some issues that ETX does not handle. ETT is the expected transmission time needed to successfully deliver a packet along a path. ETT value of the path is the summation of each links' ETT along the path. The formula to compute ETT is given as:

$$
E T T=E T X \times \frac{S}{B}
$$

ETT takes the bandwidth and packet size into account. In the above formula, $\mathrm{S}$ denotes the packet size and B is current link bandwidth. ETT uses a packet pairs scheme [43] to estimate link bandwidth. Nodes send two back-to-back probe packets to each neighbor node periodically, initially a small packet (137 bytes) followed by a large one (1137 bytes) [42] 
[44]. Neighboring nodes record the inter-arrival time between two probe packets and report to the sender. After receiving a number of consecutive samples, the sender divides the larger size probe packet by the smallest sample to estimate the link bandwidth.

Additional to the properties of ETX, ETT captures link capacity. This increases the individual paths throughput and hence, collectively improves overall performance of the network. Similar to ETX, ETT is also isotonic.

However, ETT retains many disadvantages of ETX. ETX does not consider link load, which makes it hard to avoid a flow through heavily loaded nodes and links. ETT was not designed for multi-radio and multi-channel networks, so it does not account for intra-flow interference. For example, ETT will select a path with one channel, but not consider a better path with more channel diversity that might lead to less intra-flow interference and higher throughput.

\subsubsection{WCETT}

Weighted Cumulative Expected Transmission Time (WCETT) [42] is an extension metric of ETT, which considers channel diversity to reduce intraflow interference. The formula of WCETT is as given below:

$$
W C E T T=(1-\beta) * \sum_{i=1}^{n} E T T_{i}+\beta * \max _{1 \leq j \leq k} X_{j}
$$

The formula of WCETT contains two parts: one is the sum of link ETT values on a path, in other words the summation of transmission time; and the second part is the summation of ETT link values of bottleneck channels along a path, which impacts the throughput of this path. $X_{j}$ means channel $j$ stands for the bottleneck channel.

WCETT uses the value $\beta$ as a tunable parameter, which is anywhere between 0 to 1 . The value of $\beta$ is used to decide different proportion of weights for the total sum of link ETT along the path and the channel diversity, which in other words is a tradeoff between path delay and through- 
put. Consider two extreme values of $\beta$. When $\beta=0$, WCETT equals to ETT, which means it does not consider channel diversity. When $\beta=1$, it will result in more hops with non-bottleneck channel and will not affect the metric value. But it cannot be true, as the bottleneck channel will dictate the total path throughput always. From this formula, we know that channel diversity is very important, especially for a short path. This is because when considering more hops, the summation of ETT increases rapidly, but not channel diversity.

WCETT is based on ETT, so it inherits all the properties of ETT and ETX. The primary improvement of WCETT is that it addresses intra-flow interference by considering channel diversity. It gives lower weight to the path with more diversified channels assigned to their links, resulting in lower intra-flow interference. In short, WCETT is more suitable for multiradio and multi-channel networks.

The disadvantages of WCETT are obvious. First, WCETT considers the number of links using the same channel and their respective ETTs, but without involving the link location [32]. For example, if two links operate on the same channel but are out of each other's interference range, they will not reduce the throughput. WCETT ignores this situation, and considers that all links operate on the same channel and interfere with one another. As a consequence, the smallest weight path selected by WCETT may not be correct.

Secondly, WCETT is not isotonic, which means it does not work well for link state routing protocols that use algorithms like Dijkstra's. . This is because these algorithms calculate the minimum weight path by adding each links' metric value hop by hop. So the minimum weight path selected by WCETT may not be the same. Additionally, WCETT does not explicitly take inter- flow interference into account. This will lead to routes selected by WCETT to traverse a densely loaded path causing high level of interference and resulting in node starvation [23, 45].

Despite these shortcomings, WCETT improves network performance 
for multi-radio and multi-channel WMNs. Using WCETT we can calculate more accurate minimum weight path than hop count, ETX and ETT.

\subsubsection{MIC}

The Metric of Interference and Channel switching cost (MIC) [46] was designed to improve WCETT metric by capturing inter-flow interference. The MIC of a path $p$ is given as follows:

$$
M I C(p)=\frac{1}{N \times \min (E T T)} \sum_{\text {link } \in p} I R U_{l}+\sum_{\text {node } i \in p} C S C_{i}
$$

In formula 2.4, $N$ is the total number of nodes in the network, and $\min (E T T)$ is the smallest ETT of the entire network. IRU denotes the Interference-aware Resource Usage, and $C S C$ denotes the Channel Switching Cost. These two components are defined as follows:

$$
\begin{aligned}
& I R U_{l}=E T T_{l} \times N_{l}, \\
& C S C_{i}=\left\{\begin{array}{ll}
w_{1} & \text { ifCH }(\operatorname{prev}(i)) \neq C H(i) \\
w_{2} & \text { ifCH }(\operatorname{prev}(i))=C H(i)
\end{array} \quad 0 \leq w_{1} \leq w_{2},\right.
\end{aligned}
$$

In formula 2.5 and 2.6, $N_{l}$ is the number of neighbors that interfere with the transmission on link $l, C H(i)$ denotes the channel that node $i$ uses for transmission, and prev $(i)$ denotes the previous hop of node $i$ along the path $p$. The $I R U_{l}$ component refers to the total channel time that neighboring nodes take to transmit on link $1 . I R U_{l}$ captures inter-flow interference by calculating the channel time. Lower $I R U_{l}$ values represent lower levels of inter-flow interference. The $C S C$ component captures the intra-flow interference since consecutive links using same channel will have higher weights than the paths with more diversified channel assignments.

Although MIC captures intra-flow and inter-flow interference, it still has some limitations. First, the $C S C$ component only considers the situation of two consecutive links for capturing intra-flow interference [47]. 
If there is a link with different channels between two links using same channel and within each other's interference range, there is still intra-flow interference.

Another limitation of MIC is that the IRU component does not account for the fact that the degree of interference caused by neighboring nodes is not the same [45]. Different degrees of interference may result in varied signal strengths based on the interfering nodes or the positions of the interferers. For example, consider two links that have the same ETT value, one node may have two interfering neighbors which are close to the link in question and cause high degree of inter-flow interference, and another node has three interfering neighbors which have much lower inter-flow interference. MIC will incorrectly choose the first link with poor throughputs. Another drawback of the $I R U$ component is that it may lead to MIC choosing the path with fewer neighbors, resulting in longer and slower paths.

The third limitation is the MIC metric is not isotonic, because of the $C S C$ component.For example, the channel of a third path may be the same as one of two previous paths, and might causes MIC value to change. To address this, Yang, et al. [23] presents a more complicated method in which nodes can be considered as virtual in order to make the metric isotonic. MIC also does not consider load balancing, as the overhead caused by maintaining update information of ETT for each link will differ and affect performance unfairly throughout the network.

\subsubsection{Summary}

From the analysis above, we can conclude that a good metric for WMNs should meet certain criteria. As these metrics have been designed to perform well for different routing protocols, algorithms or conditions, it is not fair to expect every metric to satisfy all the criteria. Various metrics have been developed as a direct improvement to its predecessor in terms of one 
specific criterion and hence might fail to satisfy other criteria.

The hop count metric ignores interference, link quality, link capacity, and channel diversity. As a consequence, hop count has limited use in WMNs, especially in multi-radio and multi-channel WMNs. However, it is simple and provides a high level of stability and has the isotonicity property, which allows it to find minimum weight paths efficiently.

The ETX metric is designed to account for link quality. It chooses a path entirely based on link quality ignoring hop count. However, ETX also does not consider link capacity and does not capture interference; thus, it only works better in multi-radio and multi-channel WMNs. ETX uses a probing method to estimate link quality, but it may not be accurate when there is conflicting traffic. Also, ETX does not consider link load and cannot perform network load balancing. ETX is isotonic, so it works well with certain link state algorithms to calculate minimum weight path.

The ETT metric is built to extend ETX by considering link capacity. Except for capturing link capacity, ETT retains almost all advantages and disadvantages of ETX such as, ignorance to interference, especially intraflow interference. Hence, ETT is not suitable for multi-radio WMNs.

The WCETT metric improves ETT by considering intra-flow interference, and retains many characteristics of ETT. It captures intra-flow interference by considering channel diversity, hence WCETT improves throughput and is more suitable for multi-radio and multi-channel WMNs. However, WCETT is not isotonic, and this results in it being hard to use with link state routing protocols. Also, WCETT does not explicitly take interflow interference into account, so it may suffer from high levels of interference.

Finally, MIC attempts to overcome the limitations of WCETT by directly considering inter-flow and intra-flow interference. But, due to its $C S C$ component (discussed above), it does not fully consider all the situations of intra-flow interference. MIC is also not isotonic and does not consider link load, but it still performs well in multi-radio and multi- channel 
radio WMNs. Table 2.1 shows which of the criteria identified in Chapter 2.2 are met by different metrics. In next chapter we will describe the modification of OLSRv2 for implementing the ETX and ETT metrics.

\begin{tabular}{|l|c|c|c|c|c|}
\hline & Hop Count & ETX & ETT & WCETT & MIC \\
\hline Intra-flow Interference & $\sqrt{ }$ & $\times$ & $\times$ & $\sqrt{ }$ & $\sqrt{ }$ \\
\hline Inter-flow Interference & $\times$ & $\sqrt{ }$ & $\sqrt{ }$ & $\sqrt{ }$ & $\sqrt{ }$ \\
\hline Load Balancing & $\times$ & $\times$ & $\times$ & $\sqrt{ }$ & $\times$ \\
\hline Isotonicity & $\sqrt{ }$ & $\sqrt{ }$ & $\sqrt{ }$ & $\times$ & $\times$ \\
\hline Locality of Information & $\times$ & $\times$ & $\times$ & $\times$ & $\times$ \\
\hline Stability & $\sqrt{ }$ & $\times$ & $\times$ & $\times$ & $\times$ \\
\hline
\end{tabular}

Table 2.1: Metric Characteristics 


\section{Chapter 3}

\section{Modification of OLSRv2}

\subsection{Introduction}

In this chapter, the modification of OLSRv2 and implementation of ETX and ETT are described. The major section of the modification is focused on MPR algorithm. As we know, the original MPR algorithm is based on hop count. When we use link quality metrics, the original MPR algorithm will still result in some poor quality links. This is because the original MPR algorithm does not take the best 2-hop paths into account. The modification in the OLSRv2 module solves this problem. Some simple examples of MPR selection are shown for better understanding of the modification.

\subsection{Implementation of the ETX Metric}

ETX is a metric that measures link quality by calculating the probability of packet loss by exchanging periodic control messages between neighbors, which uses the message format defined in RFC 5444 [48]. The calculation of ETX metric is only based on network layer signaling. If we use the ETX metric instead of hop count in OLSRv2, two main aspects should be considered, which are periodically broadcast probes to get the packet loss 
ratio and modification of MPR algorithm. The OLSRv2 module used for our simulation is developed by Niigata University OLSR research group, and the module can be downloaded from Niigata research website [49].

\subsubsection{Modification of the Hello message}

To get the value of the ETX metric, every node needs to broadcast a probe packet to all its 1-hop neighbors periodically. Adding probes in OLSRv2 causes additional overheads; hence the performance of the network will decrease. The module uses Hello messages instead of probes to calculate the delivery ratio. This is because, similar to probe packets, the Hello message is broadcast to all its 1-hop neighbors periodically, and additionally it is smaller in size. Hence this method avoids the additional control messages that can result in high overheads. To record how many Hello messages have been received an extra field needs to be added as part of the Hello message format.

\subsection{MPR algorithm modification}

Since the original MPR algorithm in OLSR and OLSRv2 is based on hop count, the algorithm must be modified to perform based on the link metric. In general, the new algorithm will generate more MPRs in the network, which can cover the entire links with good metric values [50]. The MPR algorithm in the the OLSRv2 module is divided into two parts: flooding MPRs and routing MPRs [51].

Before going into some examples to understand flooding and routing MPRs in the following subsections, we would like to explain some terminologies which will be used: 1) the numbers in figures below are their respective metric values of links, and the smaller values imply better link quality; 2) strict 2-hop neighbor: a 2-hop neighbor is not the node itself or a 1-hop neighbor of the node; 3 ) symmetric link: a verified bi-directional 
link between two nodes interferences; 4) symmetric 1-hop or 2-hop neighbor: the neighbor has at least one symmetric link to the node; and 5) symmetric strict 2-hop neighbor: a 2-hop neighbor of node A, which has a symmetric link to A's symmetric 1-hop neighbor [10].

\section{Flooding MPRs}

Flooding MPRs are used to forward control messages. Usually flooding MPR selection can ignore link quality metrics, because flooding MPRs are mostly concerned with covering 2-hop neighbors. However, this does not mean that link quality metrics cannot be useful.

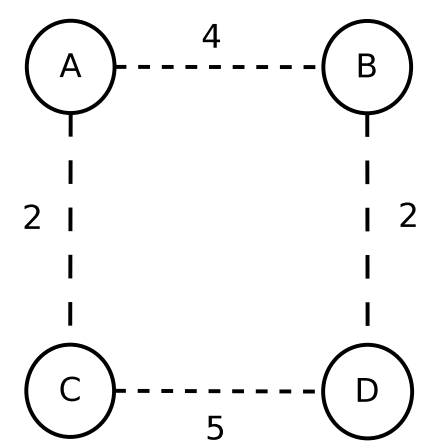

Figure 3.1: An example of selecting flooding MPRs

Consider the network in Figure 3.1, where the numbers are the metric value of links farther from node $\mathrm{A}$, towards node $\mathrm{D}$. If the metric presents the probability of packet loss similar to ETX; then choosing B as a MPR is a better choice to minimize the loss probability of a packet sent by A towards $D$, even if $C$ has a lower metric value connected to A. Similar arguments can be made for choosing B if the metric behaves like ETT or represents delay rather than the probability of loss. However, flooding MPR selection should not only consider just the first or second hop's metric value. The sum of metrics values along the 2-hop path is the correct measurement to choose a flooding MPR between B and C. 
Figure 3.1 only portrays a relatively simple example. A generic process to select a router as a flooding MPR should incorporate the metric's value to the router, and the metric's value between that router and each of its strict symmetric 2-hop neighbors, as well as the number of symmetric strict 2-hop neighbors it can cover.

The flooding MPR algorithm of OLSRv2 chosen in this simulation, first considering the 1-hop neighbors that have the largest coverage of symmetric strict 2-hop neighbors. If more than one router has the same coverage, then choosing the one that has the smallest sum of metric value of first hop and second hop.

\section{Routing MPRs}

When using a link quality metric rather than the hop count metric, it is sufficient that routing MPRs provide not only a 2-hop route, but also a minimum distance of a 2-hop route. To obtain all the good links, the symmetric strict 2-hop neighbors need to be adjusted. For routing MPRs selection, a router is a symmetric 1-hop neighbor, but there is a 2-hop path to it that is shorter (smaller metric value) than the 1-hop path. This router needs to be considered as a symmetric strict 2-hop neighbor for routing MPR selection.

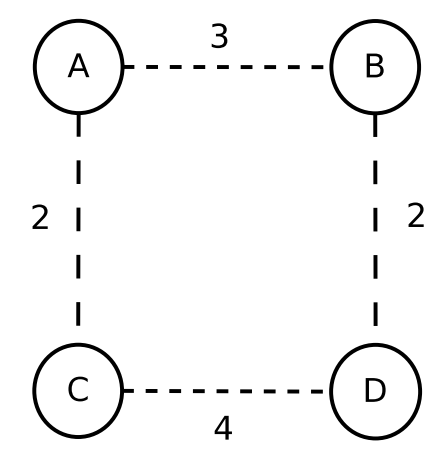

Figure 3.2: Routing MPRs selection examples

In Figure 3.2, for minimum hop count routing, router A can pick either 
$B$ or $C$ as MPRs. But when using link metrics, $A$ must pick $B$ as a routing MPR, which can provide a better quality link than the other one.

In Figure 3.3, for the minimum hop count routing, router A does not need to select a routing MPR. When using link metrics, A must pick router $B$ as its routing MPR, which will result in a better link quality path from $B$ to $C$ rather than from A directly to $C$.

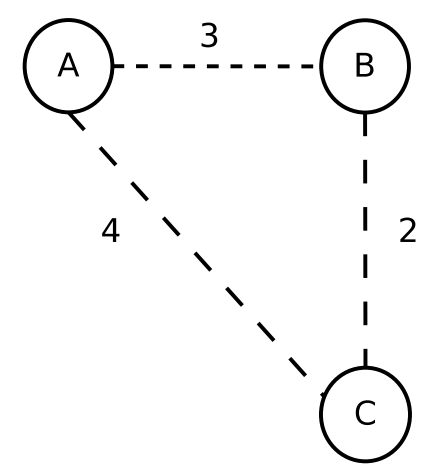

Figure 3.3: Routing MPRs selection examples

Consider the network in Figure 3.4. For the minimum hop count routing, router A only needs to choose one between $B$ and $C$ to be its routing MPR as either of the two can cover 2-hop neighbors. For choosing the best quality path to $\mathrm{D}$ and $\mathrm{E}, \mathrm{A}$ must pick both $\mathrm{B}$ and $\mathrm{C}$ as routing MPRs. This is because only $B$ can provide the best quality path to $D$, and only $C$ can provide the best quality path to $\mathrm{E}$.

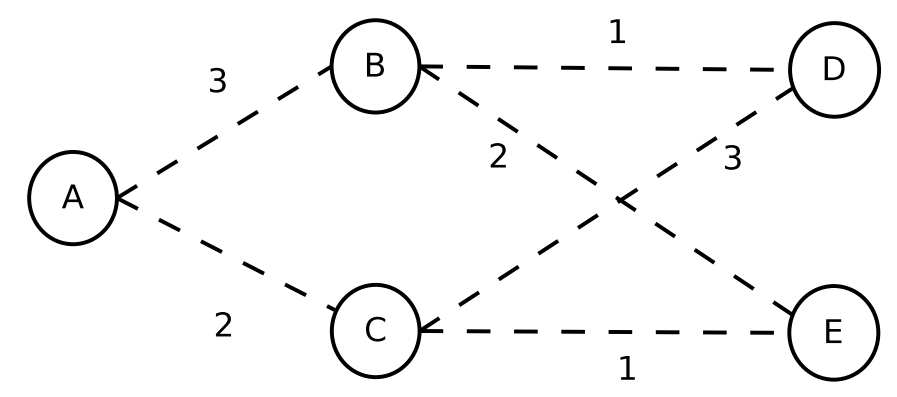

Figure 3.4: Routing MPRs selection examples [51] 
As we have mentioned above, if a router can be both a symmetric 2hop neighbor with a better two hop path and a symmetric 1-hop neighbor with a worse one hop path, we need to consider it only as a symmetric strict 2-hop neighbor. Consider the network in Figure 3.5. During MPR selection (as described above), router A must pick B and C as its routing MPRs to cover all the symmetric strict 2-hop neighbors for the following reasons: for $B$ because, the route through $B$ to $C$ with smaller weight is better than $A$ to $C$; for $C$ because, $C$ can cover A's 2-hop neighbor $D$.

However, A should not pick $C$ as its routing MPR as the other nodes need to be aware that there is only one route to A, which is through B. Consider the case when router $E$ has to find a route to A. If A selects $C$ as its routing MPR, then $E$ will be aware that there is a route from $C$ to $A$. This might lead to E choosing the route through $\mathrm{C}$. However, the route to $\mathrm{A}$ through B will be better than through $C$, as the link quality is better along the route. Hence, A should not pick $C$ as its routing MPR and therefore, should avoid advertising the link from $\mathrm{C}$ to $\mathrm{A}$.

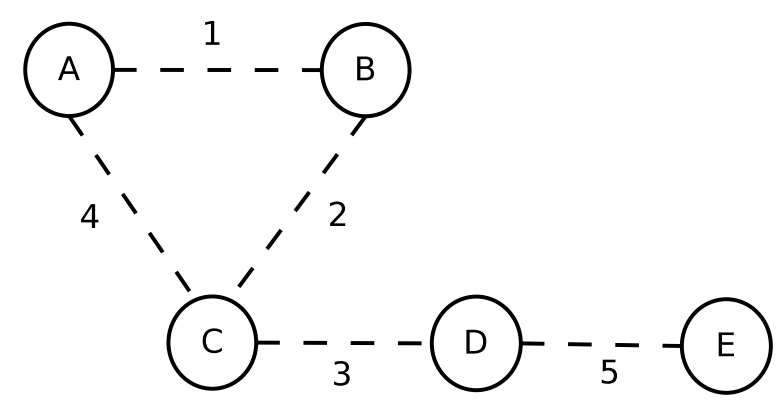

Figure 3.5: Routing MPRs selection examples [51]

From the above modification of routing MPRs, using link metrics may require a router to select more routing MPRs than using hop count. Without link metrics, a router does not even have to select any routing MPRs. Selection routing MPRs results in more and larger control message been generated and forwarded to the entire network. This is the cost of using link metrics. More control messages and more overheads can not be 
avoided.

\section{Relationship between Flooding MPRs and Routing MPRs}

If flooding and routing MPR sets are the same, no additional control messages and overheads will be generated. This will happen only when all the link metric values are equal (MPR selection behaves such that the selection is based on hop count), but this situation cannot occur, especially when there are multiple interfaces and the metric considers the direction of the link. Although, we consider only one interface on each node in our scenarios, the flooding and routing MPR sets are not equal nor is one MPR set a subset of the other.

In Figure 3.6, router A does not need to select any flooding MPRs, because all the neighbors are symmetric strict 1-hop neighbors. However, A must select $B$ as a routing MPR, because $C$ needs to be considered as a symmetric strict 2-hop neighbor that has a better route through $B$.

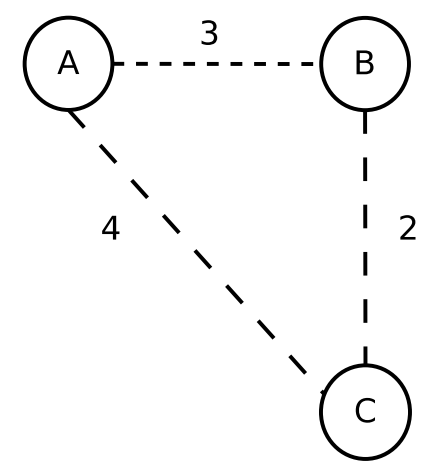

Figure 3.6: Selecting routing and flooding MPR sets

In Figure 3.7, the minimal flooding MPR set of router A is B (as B can cover all 2-hop neighbors), but $A$ must select $D$ and $C$ as routing MPR sets. In this case, routing MPRs, $C$ and D will serve as flooding MPRs. They provide a better route to all 2-hop neighbors compared to $\mathrm{B}$, although the flooding MPR set is not minimal. 


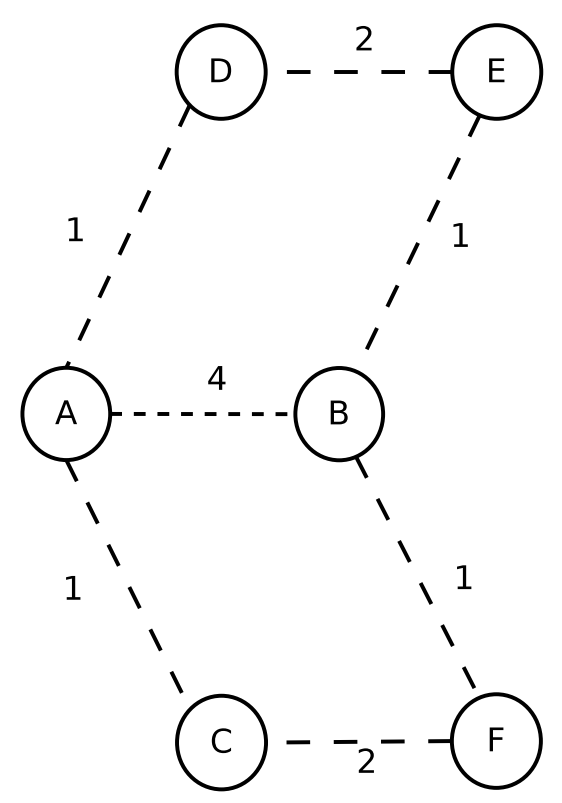

Figure 3.7: Selecting routing and flooding MPR sets [51]

However, the most common case is that the flooding MPR set and the routing MPR sets are not equal. Consider the network in Figure 3.8. C must choose $\mathrm{B}$ as a flooding MPR, but does not need to select $\mathrm{A}$. $\mathrm{C}$ must select $A$ as its routing MPR, but does not need to select $B$. In general, when using link quality metrics, a network must select minimal flooding MPR sets to cover all the 2-hop neighbors of each node and select routing MPR sets to cover all the best two hop routes.

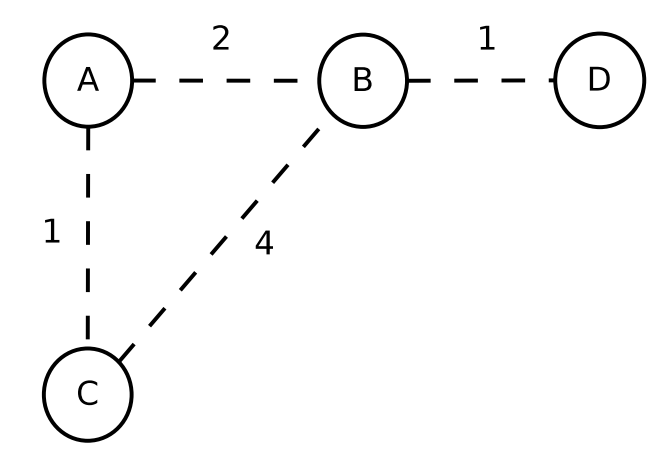

Figure 3.8: Selecting routing and flooding MPR sets [51] 


\subsection{Implementation of the ETT metric}

We use packet pairs scheme to estimate the current link bandwidth. The packet pair technique requires each node to send two back-to-back probes to each neighbor as unicast transmissions. Probes should not be sent by broadcast, as it utilizes the base IEEE 802.11 physical rate and cannot accurately estimate the current link capacity. Hence, we unicast probes at the data rate of $11 \mathrm{mbps}$.

Since the characteristics of ETT and ETX are similar, such as isotonicity, the MPR and routing algorithm do not need to be modified.

\subsection{Summary}

Using Hello messages instead of probes in the ETX metric, we avoided extra overheads that can affect the performance of the network. Also, by sending two back-to-back probes as unicast at a specific data rate, we are able to accurately estimate the link bandwidth for the ETT metric. However, there are still limitations in the implementation of ETX and ETT. In the case of the ETX metric, although we avoided excess overheads, using broadcast rates we cannot obtain the accurate link delivery ratio. Also, using small hello messages in OLSRv2 where data packets are usually larger in size will also affect the accuracy of the estimation. In the implementation of the ETT metric, the additional unicast probes will cause more overhead than the ETX metric.

When using hop count metric, the protocol only needs to select MPRs for minimal flooding to cover all its 2-hop neighbors. This is sufficient for calculating the shortest path and there is no necessity to select routing MPRs. However, when using link quality metrics, flooding MPRs' priority is to cover the 2-hop neighbors, and routing MPRs still must be selected to obtain all the best 2-hop routes. As a consequence, more MPRs result in more overheads. This essentially is the tradeoff of using link quality 
metrics to enhance link quality. The next chapter presents the simulation results and discusses the impact brought about by the metrics in different network conditions. 


\section{Chapter 4}

\section{A Grid Mesh Network Scenario}

\subsection{Introduction}

Before considering a realistic network topology, we set up a grid topology to run simulations aiming to understand certain characteristics of the three metrics. This chapter describes the performance study of the metrics under a number of different conditions; a) the impact of the path length; b) the impact of flow rate changes; and c) the impact of increases in node density.

\subsection{Simulation tool}

QualNet [52] was used as the simulation tool. This is a high-fidelity network simulator for evaluating wireless, wired, mixed-platform network, and networking device performance. It provides numerous wireless libraries such as WiFi, sensor networks, MANET, WiMAX, and satellite, that can used to set up different scenarios. 


\subsection{A Grid Topology}

Figure 4.1 shows the grid network topology; it consists of 121 static nodes placed in a $1500 \mathrm{~m} \times 1500 \mathrm{~m}$ area. The distance between each node is $140 \mathrm{~m}$, and we used CBR (Constant Bit Rate) as the traffic generator in this scenario.

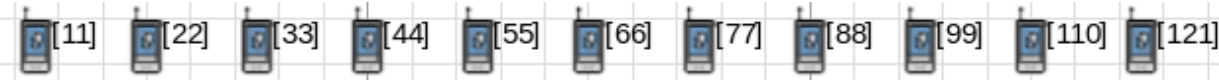

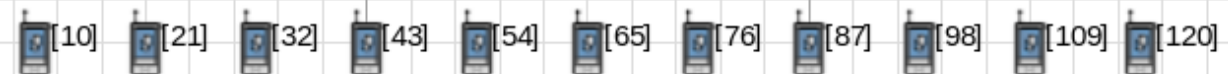

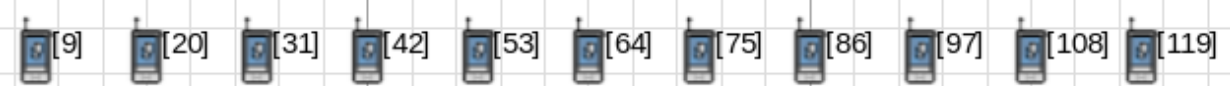

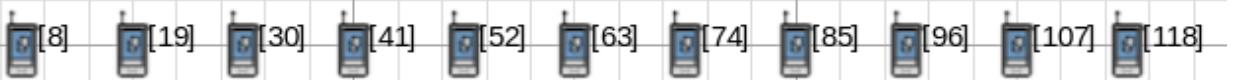

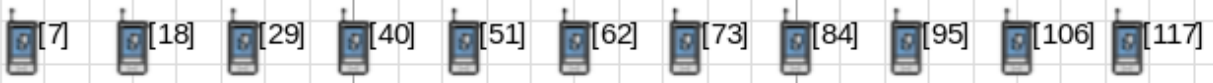

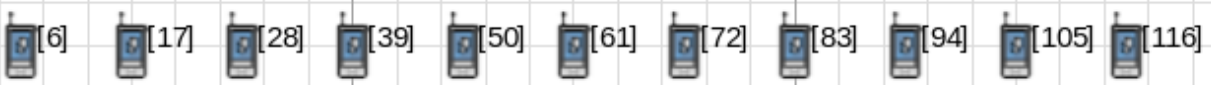

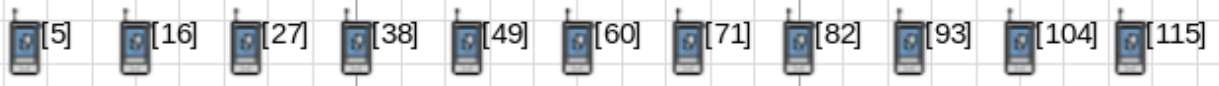

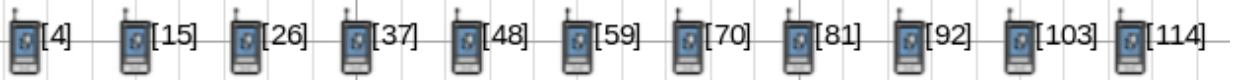

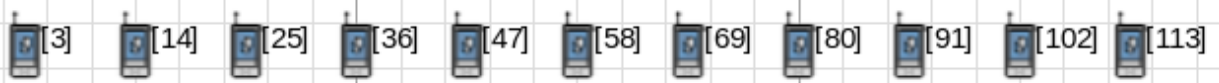

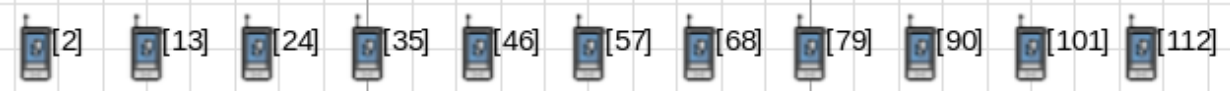

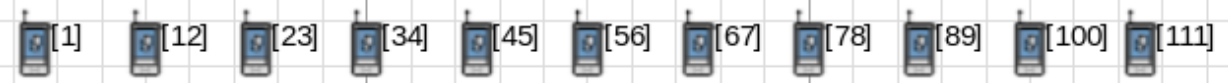

Figure 4.1: A 121 nodes grid network topology 


\subsection{Simulation Environment}

The transmitting power of each node was set to $15 \mathrm{~dB}$ and the channel frequency to $2.4 \mathrm{GHz}$. Every node was equipped with one $802.11 \mathrm{~b}$ radio and transmitted data over a single channel. As all the nodes were static, Constant Shadowing and Two Ray path loss models were used in the simulation. The antenna model was omni-directional. The various simulation parameters' values are summarized in Table 4.1 .

\begin{tabular}{|l|c|}
\hline Simulation Parameter & Value \\
\hline Simulation Area & $1500 \mathrm{~m} * 1500 \mathrm{~m}$ \\
\hline Number of Nodes & 121 \\
\hline Simulation Time & $300 \mathrm{~s}$ \\
\hline Physical Model & $802.11 \mathrm{~b}$ \\
\hline Channel Frequency & $2.412 \mathrm{GHz}$ \\
\hline Data Rate & $11 \mathrm{mbps}$ \\
\hline Transmission Power & $15 \mathrm{dBm}$ \\
\hline Transmission Range & $283.554 \mathrm{~m}$ \\
\hline PathLoss Model & Two-Ray \\
\hline Shadowing Model & Constant \\
\hline Antenna Model & Omni-directional \\
\hline
\end{tabular}

Table 4.1: Simulation Parameter

Table 4.2 shows the OLSRv2, ETX and ETT parameters. Starting at 1 second, hello messages were transmitted every 2 seconds. The hold time (validity period) of each hello message was set at 6 seconds. The interval and start time of TC messages was set to 5 and 1 second respectively. TC messages were set to expire after 15 seconds. The ETX value was calculated using 10 consecutive samples. Two back-to-back probes were sent every 15 seconds, and 5 consecutive samples were used for computing bandwidth. The validity period of each of these samples was 60 seconds. 


\begin{tabular}{|l|c|}
\hline OLSR and Metrics Parameter & Value \\
\hline Hello Message Interval & $2 \mathrm{~s}$ \\
\hline Hello Hold Time & $6 \mathrm{~s}$ \\
\hline TC Message Interval & $5 \mathrm{~s}$ \\
\hline TC Hold Time & $15 \mathrm{~s}$ \\
\hline ETX Window Size & 10 \\
\hline ETT Window Size & 5 \\
\hline Packet Pairs Interval & $15 \mathrm{~s}$ \\
\hline ETT expiration Time & $60 \mathrm{~s}$ \\
\hline
\end{tabular}

Table 4.2: OLSR and metrics Parameter

\subsection{Traffic Initialization}

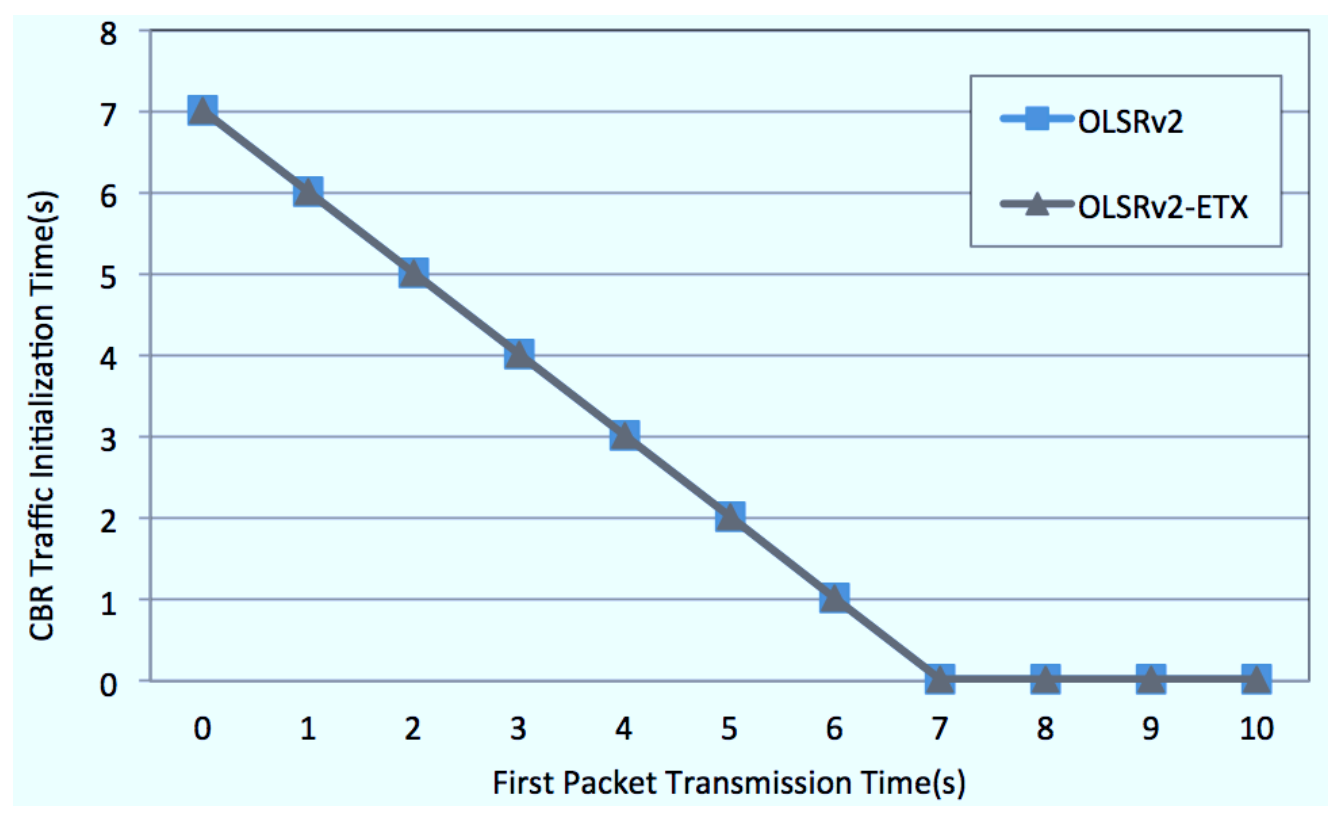

Figure 4.2: First packet transmission time

Initially, we started CBR traffic generation at 0 seconds, but found that the first packet was received only after 7 seconds. Hence, we increased the ini- 
tialization time from 0 seconds to 10 seconds to allow for the network to converge. Figure 4.2 shows the received time of the first CBR packet when the traffic initialization time was increased. The results show that the network takes about 7 seconds to converge irrespective of the routing metrics used. Following this analysis, we generated traffic from 20 seconds allowing time for nodes to complete the route discovery process and converge, therefore making the network more stable and transmission ready.

\subsection{Impact of Path Length}

There are 121 nodes in our topology, so a total of $121 \times 120$ connections are possible. Out of these, we randomly chose 50 node pairs to run the simulation. We kept tracking all the connections to get the path length, and then calculated the average path length (hops) for each metric. Figure 4.3 shows the hop count metric has the shortest path length (3.9), followed by ETX (5.2) and ETT (5.9).

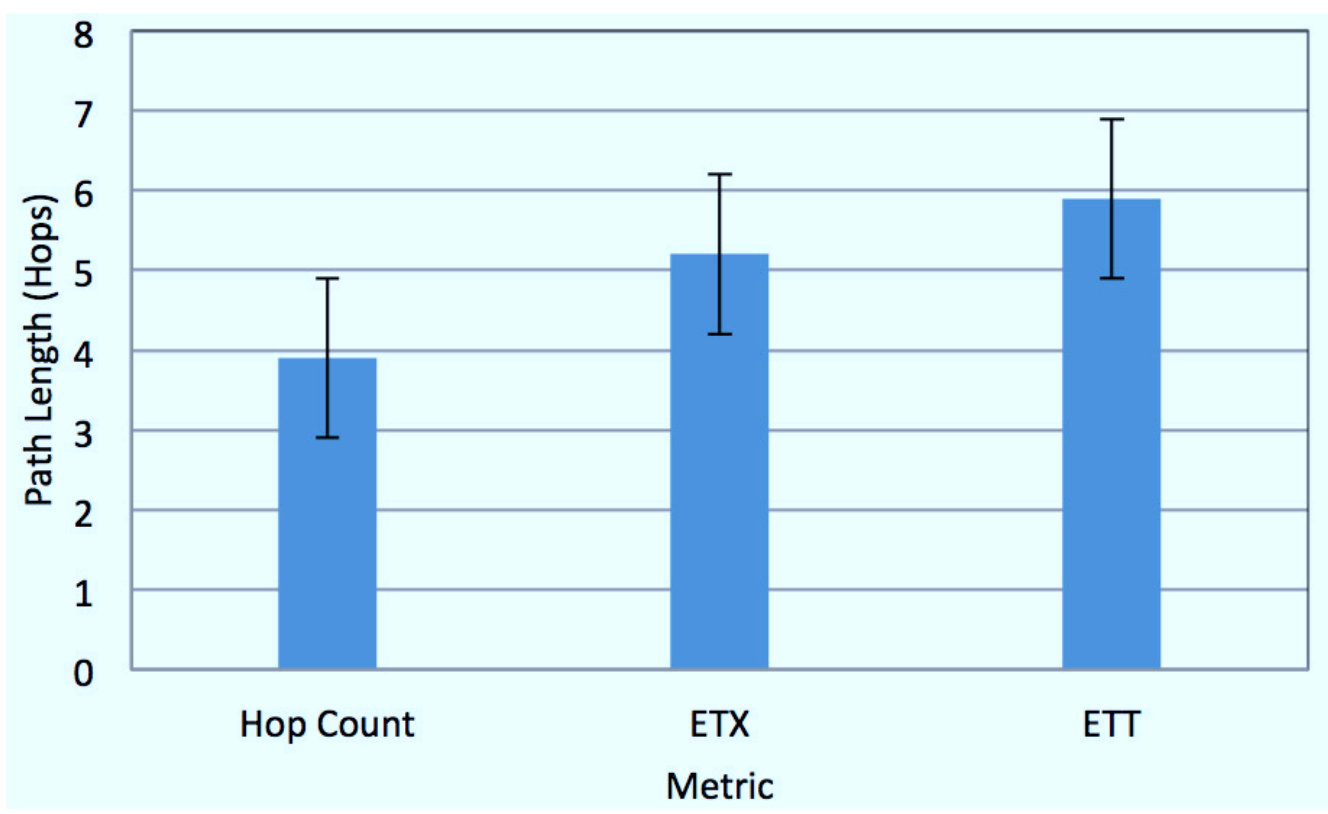

Figure 4.3: Average path length of CBR connections 
We also examined the impact of the average path length on CBR throughput. We calculated the average throughput of the connections of identical path length. Figure 4.4 plots the throughput performance when the path length is increased. As expected, shorter path length results in higher throughput. The reason being, longer paths are more likely to suffer from packet loss and interference due to increased number of wireless links. ETX and ETT provide higher throughputs than hop count by avoiding the paths with lower throughput. In particular, they performed well at longer path lengths.

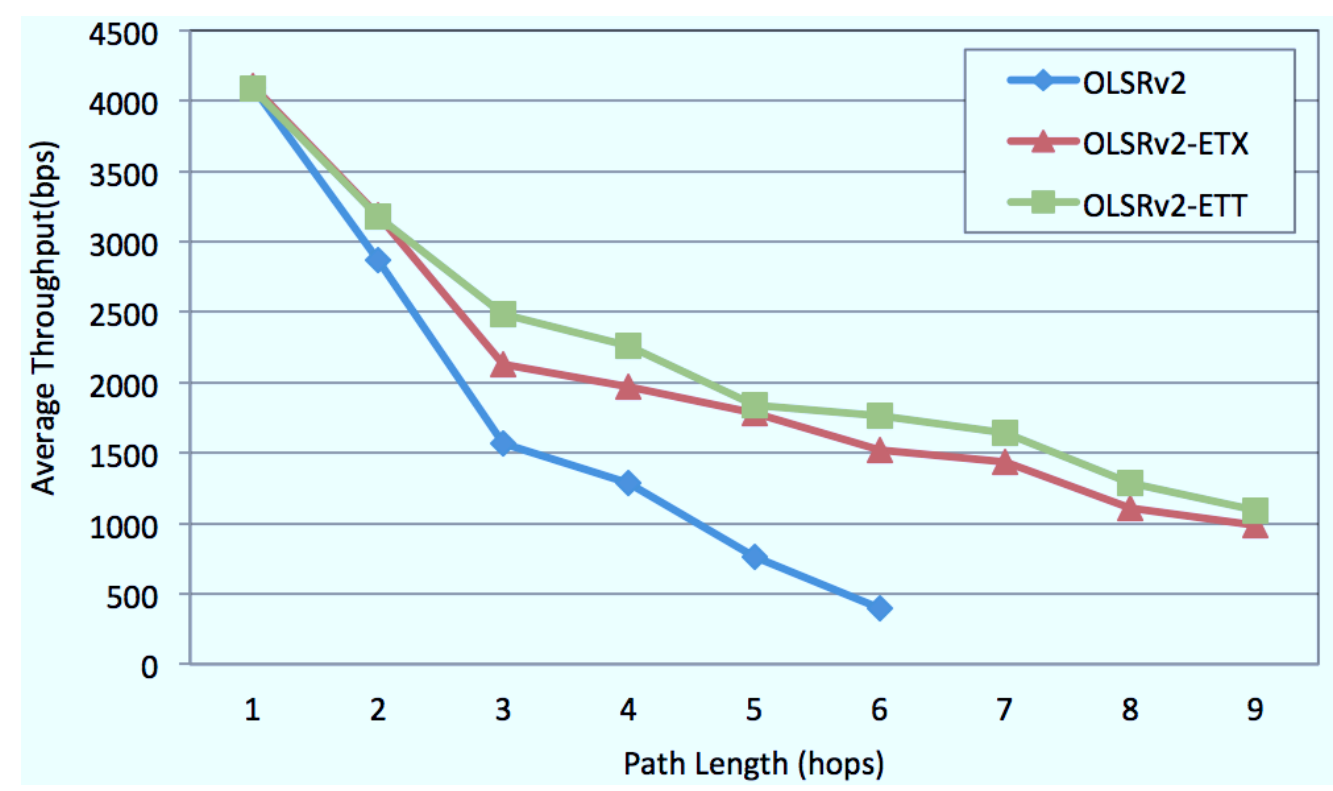

Figure 4.4: Average throughput with path length increases

\subsection{Impact of Flow Rate}

In this section, we conducted runs to study the impact of flow rate fluctuation. We increased the flow rate from 1 packet/second to 30 packets/seconds. $10 \mathrm{CBR}$ flows were initiated across the network. We cal- 
culated the average data from throughput, end-to-end delay, jitter, and packet loss ratio.

Figure 4.5, 4.6, 4.7 and 4.8 and show the performance of the three metrics under increasing flow rates. It is apparent that ETT achieves the best performance in terms of the throughput, end-to-end delay, jitter, and packet loss ratio. This is because it takes both link loss ratio and link capacity into account. Figure 4.5 supports the fact that both ETX and ETT are metrics designed to enhance throughput.

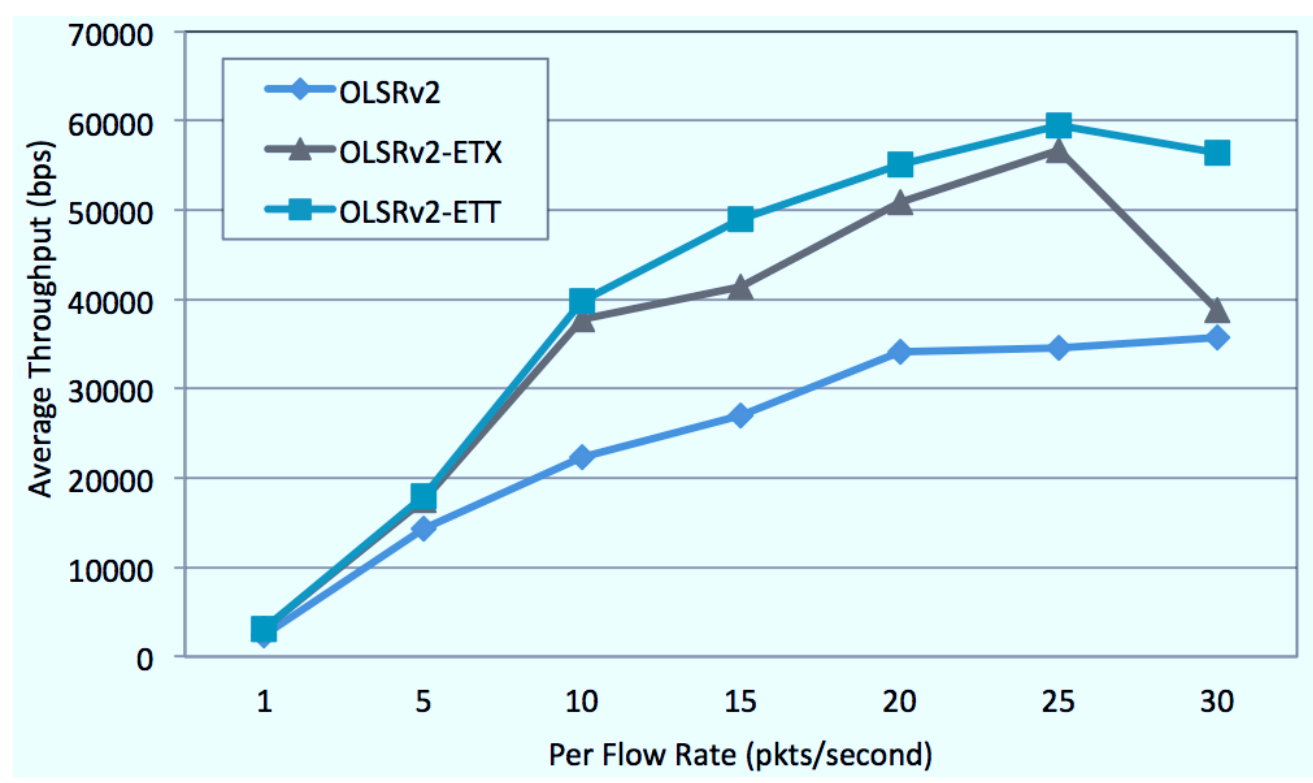

Figure 4.5: Average throughput with various CBR flow rates

As the flow rate increases, routes become more congested and the link cost will be higher. Hence, ETX and ETT will cause routes to change frequently to avoid congestion. Although both can provide higher performance than hop count, they pose a higher risk of instability due to large and irregular traffic variations. Figure 4.6 shows at low flow rates, ETX and ETT do not provide much better jitter than hop count, because when the traffic traverses through a route, the packet loss ratio will change. This causes a difference in packet loss ratio (since when it was last calculated), 
thereby making ETX and ETT recalculate weights to find a path with lower packet loss ratio. When the paths change, different path lengths cause different end-to-end delays, which results in high jitter. When the flow rate increases above 10 packets/second, the routes become more congested. This causes the jitter of hop count metric to become worse than that ETX and ETT because: 1) the packets have to wait in the queue for a longer time; and 2) as congested routes are being used, random channel contention delays become more pronounced.

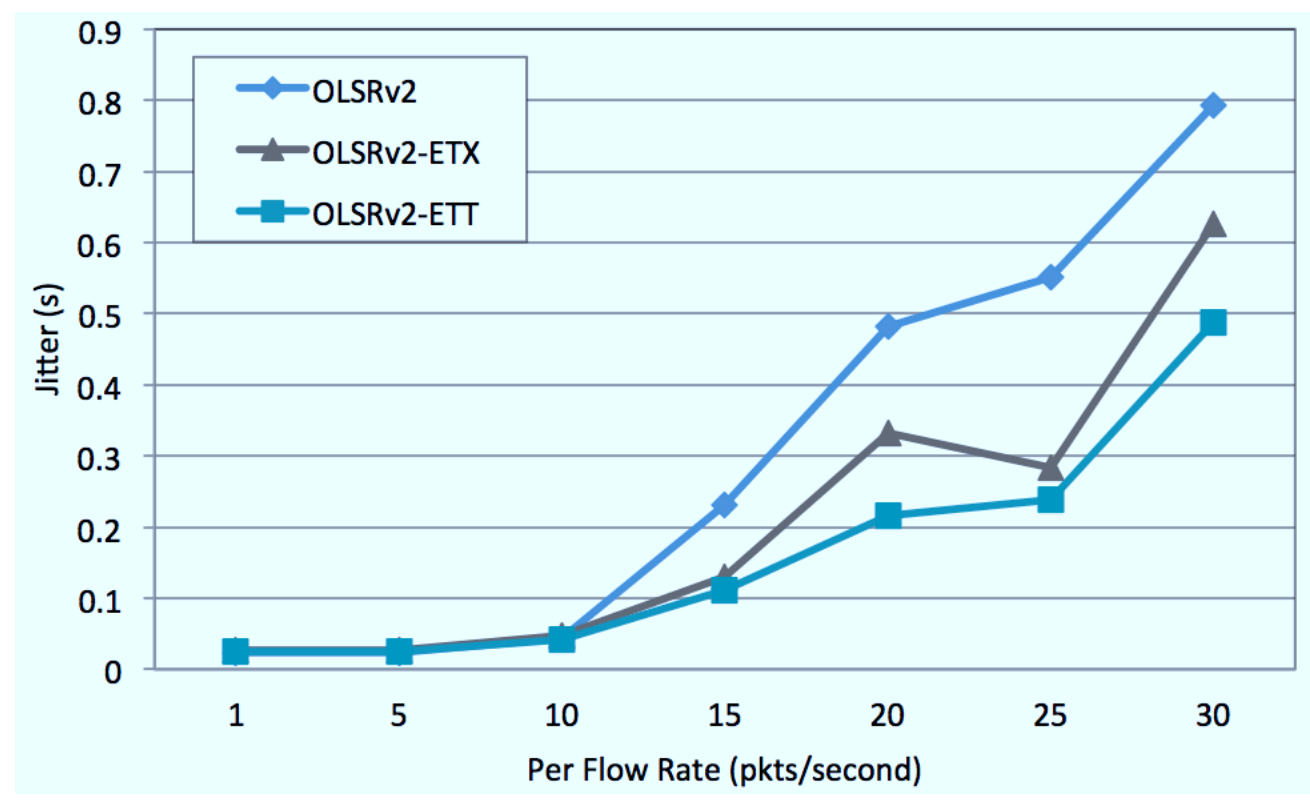

Figure 4.6: Average jitter with various CBR flow rates

From Figure 4.7, we can see that the loss ratios of the three metrics are quite high. There are two reasons for this: 1) multiple flows cause high intra-flow interference with each other; and 2) as CBR uses User Datagram Protocol (UDP) as the transport later protocol, it does not provide any reliability for data transfer. Among the three metrics, hop count shows the highest packet loss ratio for it does not weigh the links based on link quality and capacity. 


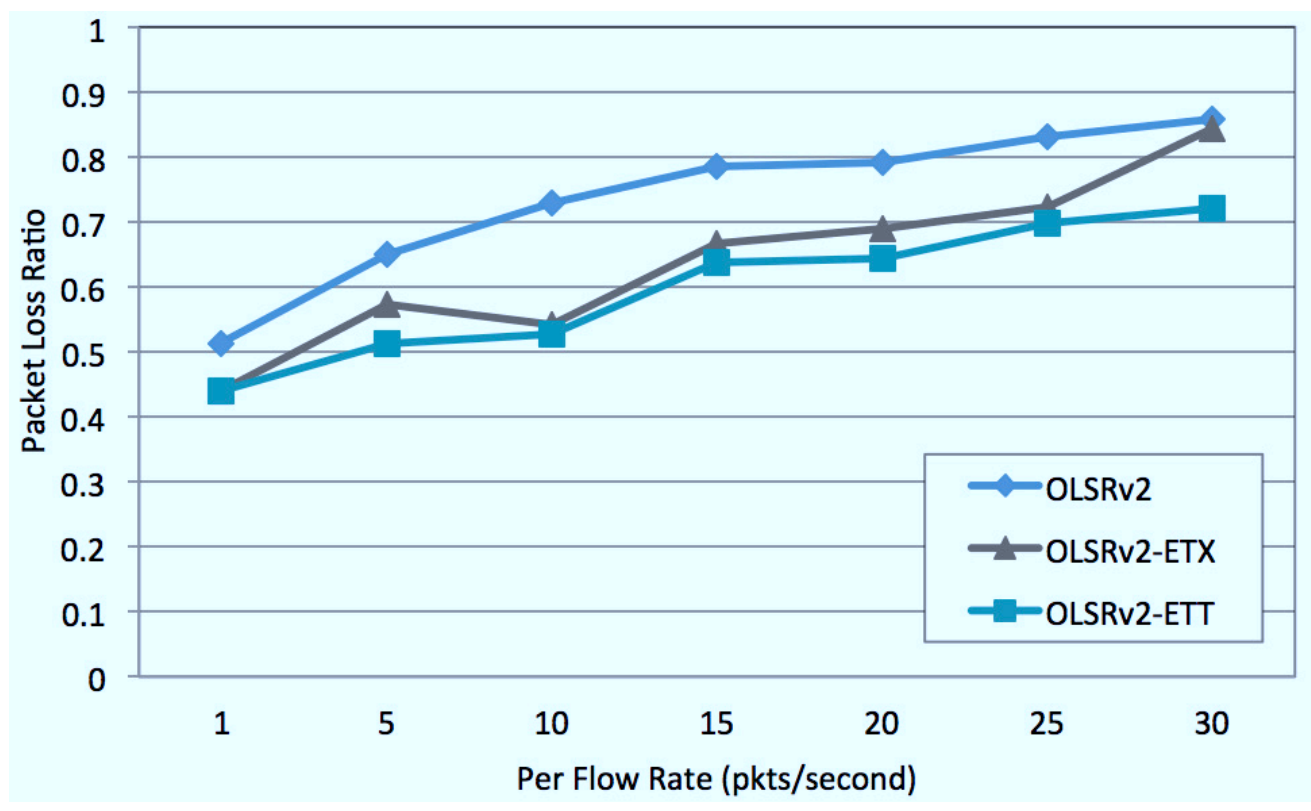

Figure 4.7: Packet loss ratio with various CBR flow rates

In Figure 4.8 , it can be seen that when the flow rate reaches 10 packets/second the end-to-end delay increases rapidly. The reason is that, when more packets are transmitted, the time spent by the packets in the queue increases, directly affecting average end-to-end delay. Figure 4.9 illustrates the average time of packets in the queue. Note that ETX and ETT have lower queuing time than hop count, because they avoid the congested paths and take a less congested route. Even though they take a longer path, lower queuing delay can still result in lower end-to-end delay.

Note that, in Figure 4.8, as the flow rate reaches 30 packets/second ETX's end-to-end delay becomes higher than hop count, and in Figure 4.7 . the packet loss ratio increases dramatically at this stage. One reason is ETX does not consider link capacity causing route changes to increase the instability of routes and aggravate packet loss, thereby affecting end-toend delay significantly. Also, the high packet loss ratio results in lower throughput, which supports the results in Figure 4.5 that is at 30 packets/second the ETX throughput is almost the same as hop count. 


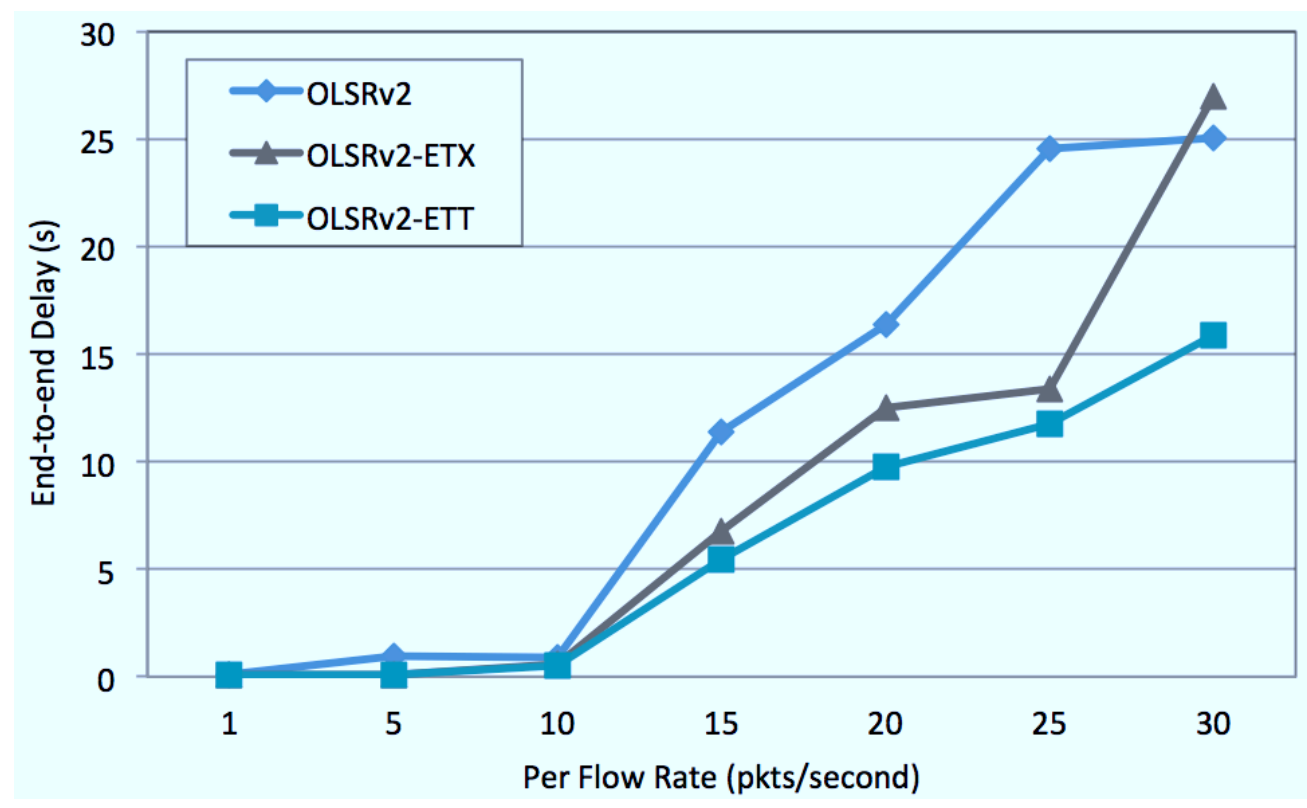

Figure 4.8: Average end-to-end delay with various CBR flow rates

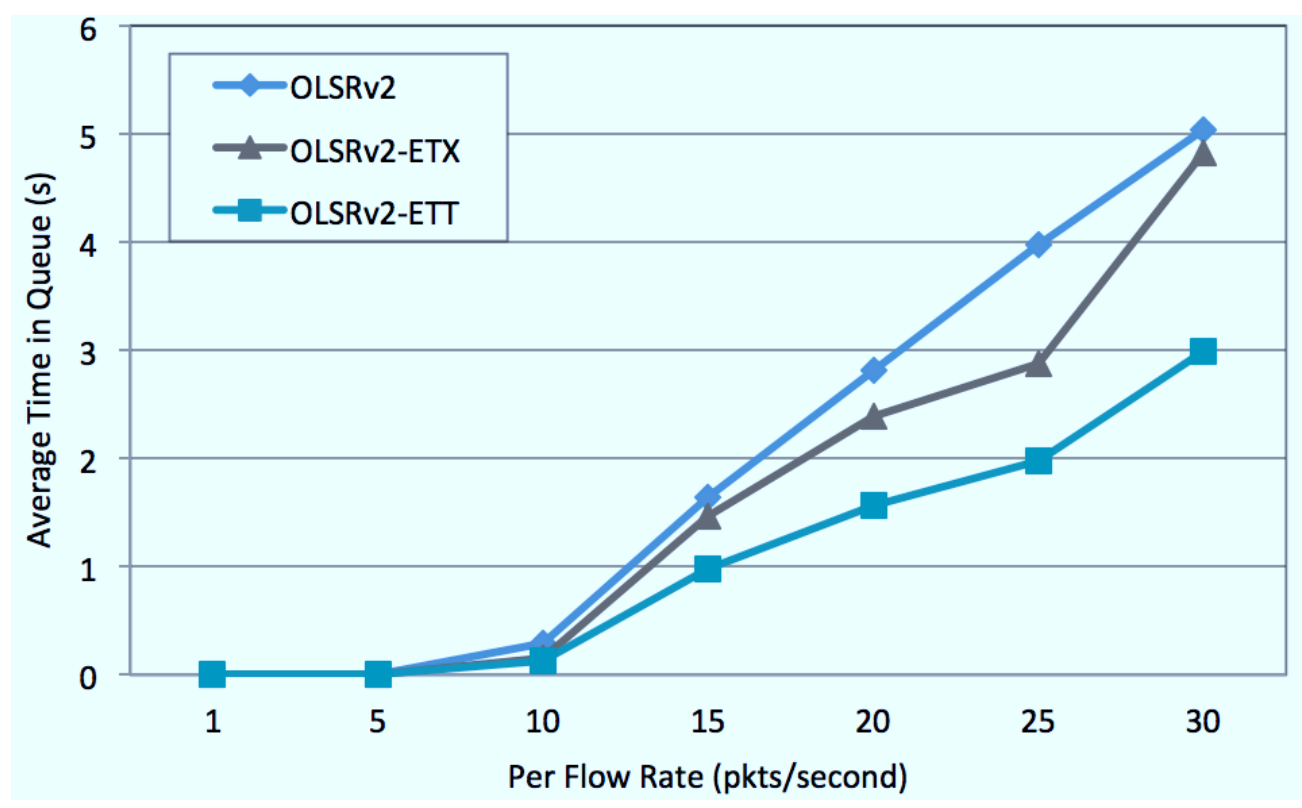

Figure 4.9: Average time of packets in queue 


\subsection{Impact of Node Density}

To evaluate the impact caused by node density, we increased the number of nodes in an area of $1500 \mathrm{~m} \times 1500 \mathrm{~m}$. Figure 4.10 shows node density increases from 50 to 250 . We used CBR as the traffic generator. $10 \mathrm{CBR}$ flows were initiated across the network. We calculated the average data for throughput, end-to-end delay, jitter, and packet loss ratio to present results.

The results are shown in Figure 4.11, 4.12, 4.13 and 4.14. In general, when the node density increased, the performance of all the three metrics was reduced, although by not too much, but ETT still outperforms ETX and ETT. The results show that the performance of hop count did not fluctuate as much as ETX and ETT. This is simply because the hop count metric always selects the path with the minimum number of hops even if the nodes are deployed densely in the network. However, denselydeployed networks for ETX and ETT mean there are more routes available between the source and the destination nodes. They can choose better quality routes to avoid congestion and reduce interference. However, the frequently changing routes cause instability of the network, which results in degradation of the network performance. Given below are some findings we need to notice:

- In Figure 4.12, ETX performs worse than hop count and ETT when the number of nodes was increased over 100. This is because hop count chooses the shortest path and is not affected by increased node density whereas ETT, which considers link capacity, provides for faster transmission than ETX.

- In Figure 4.13, the jitter of hop count is lower than ETX and ETT and does not change with the node density. This supports the fact that hop count is the least impacted by increased node density. 

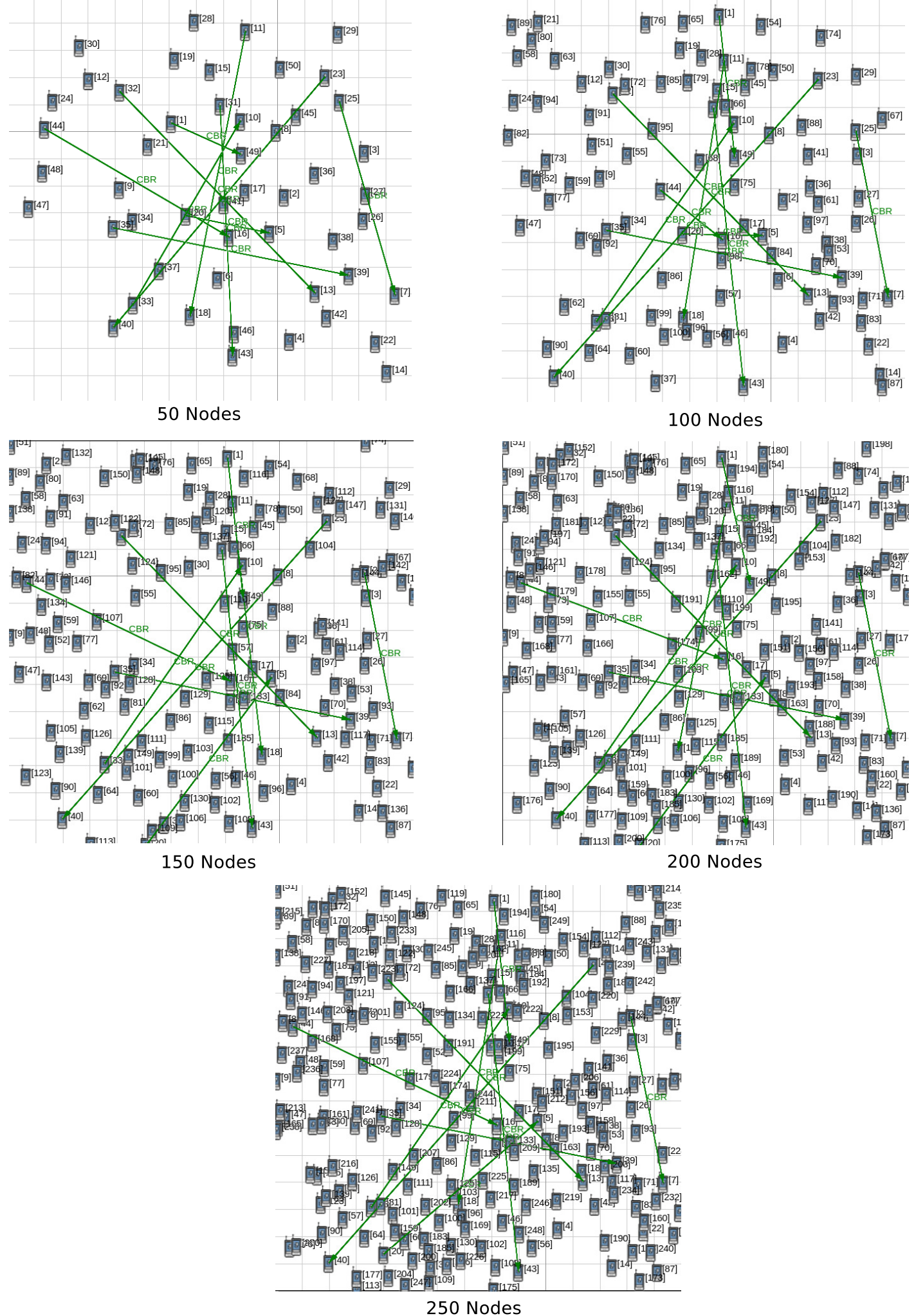

Figure 4.10: Node density from 50 to 250 


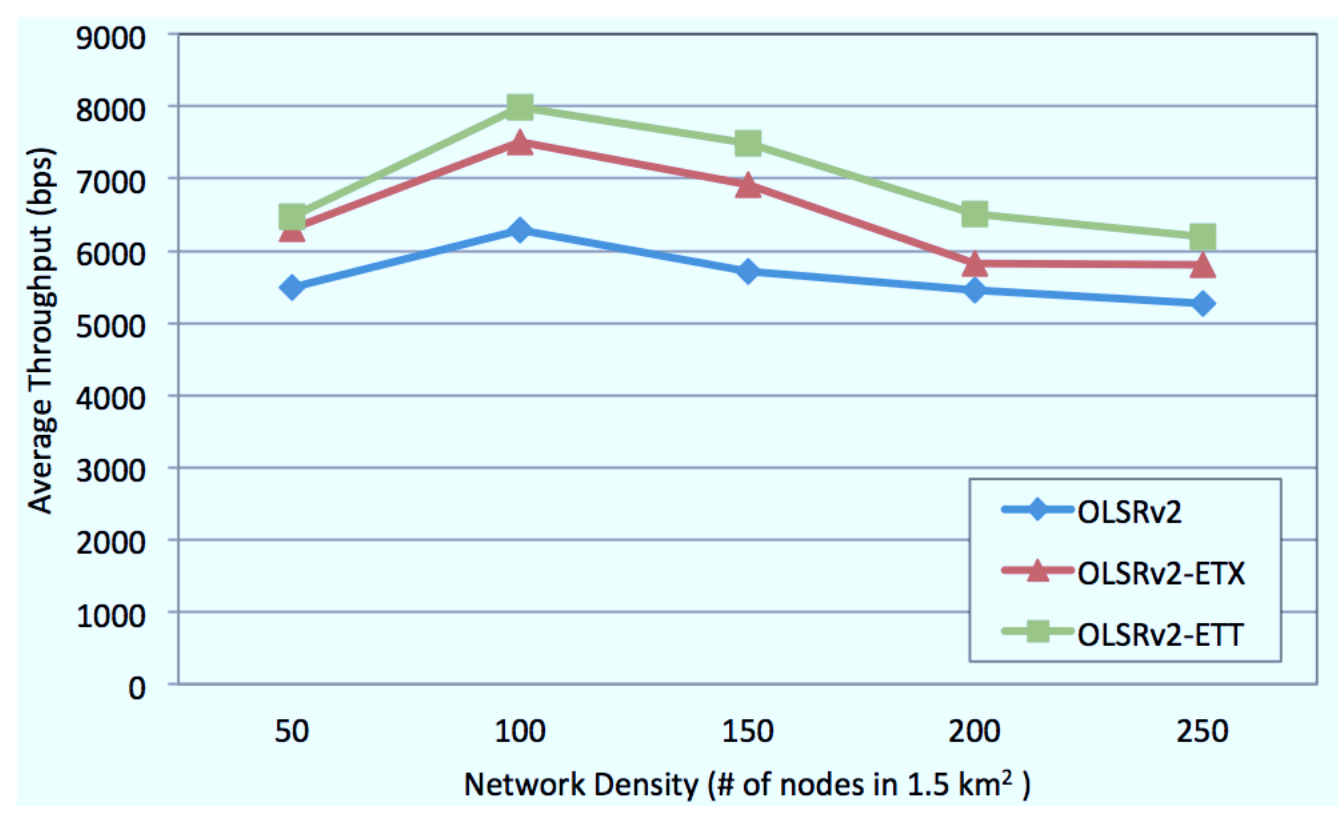

Figure 4.11: Throughput comparison with various node densities

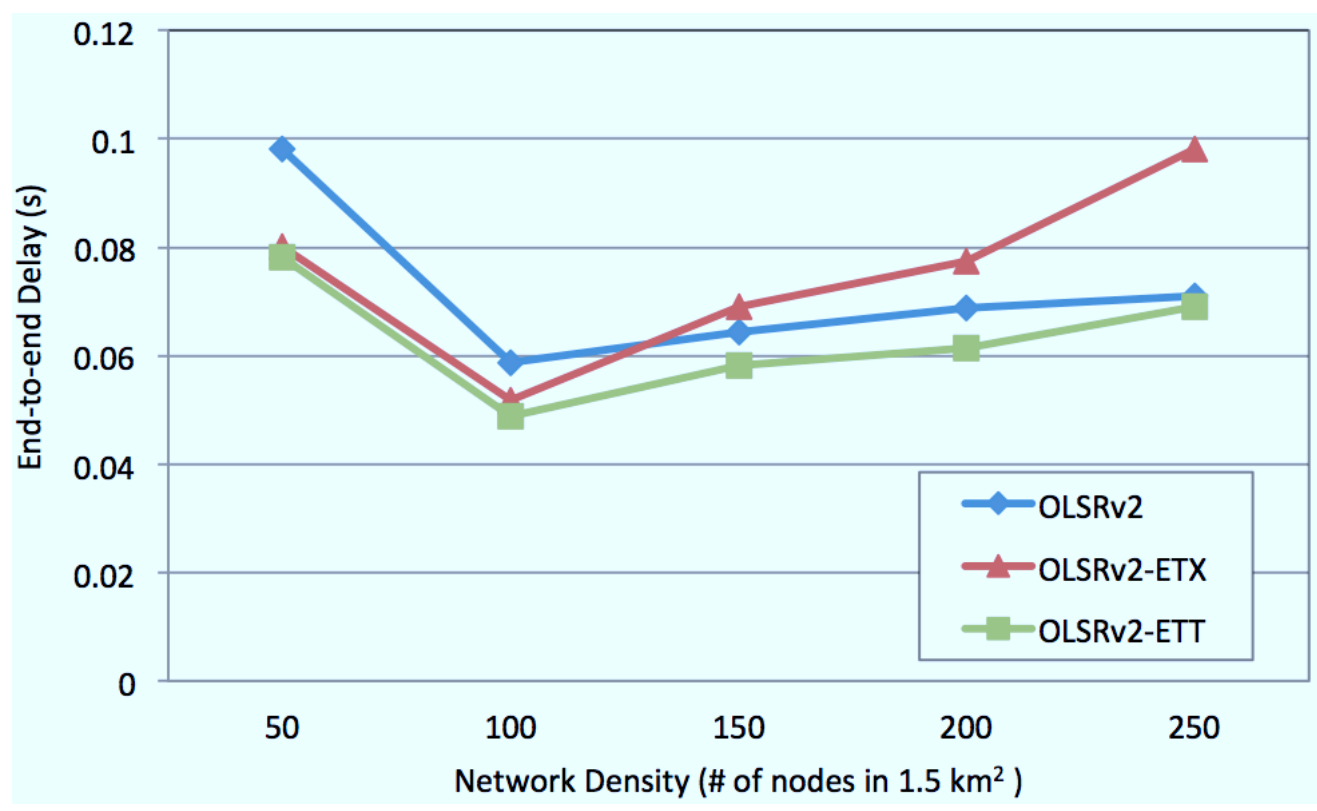

Figure 4.12: End-to-end delay comparison with various node densities 


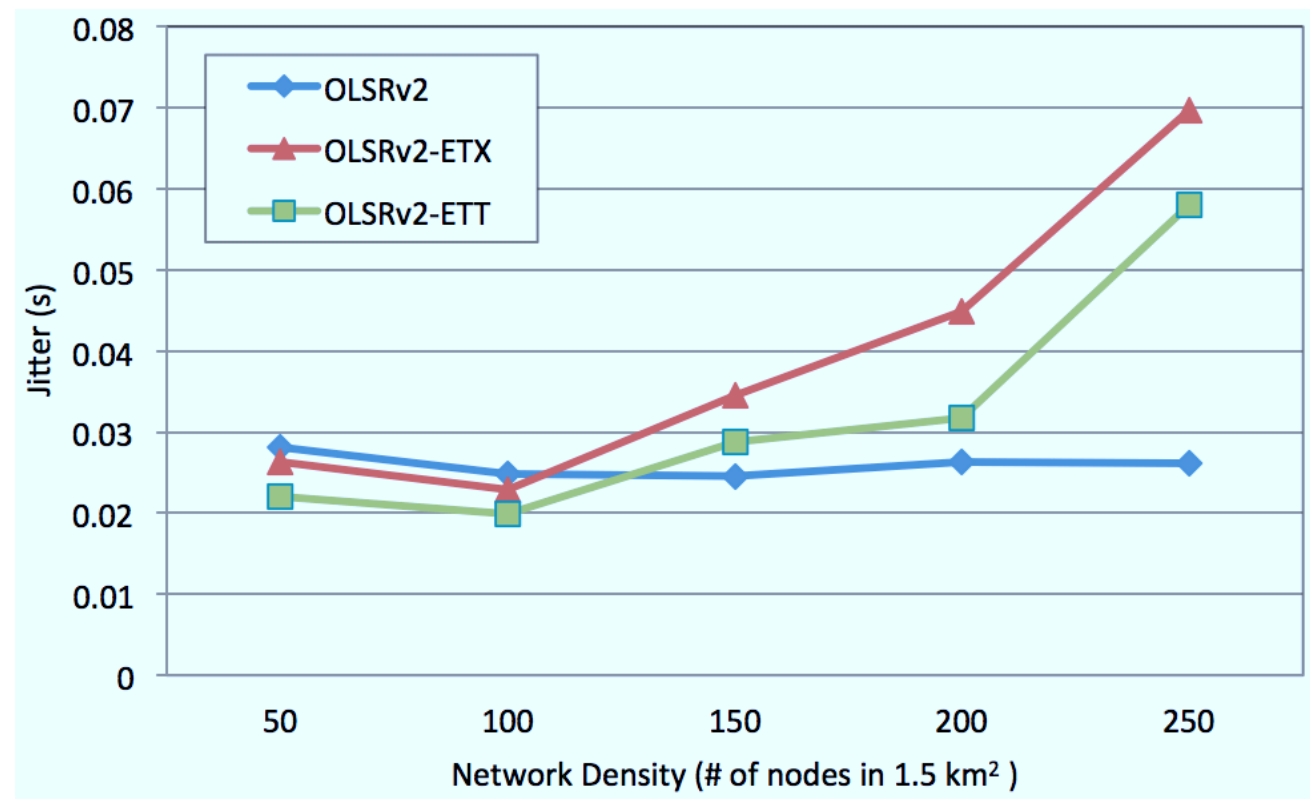

Figure 4.13: Jitter comparison with various node densities

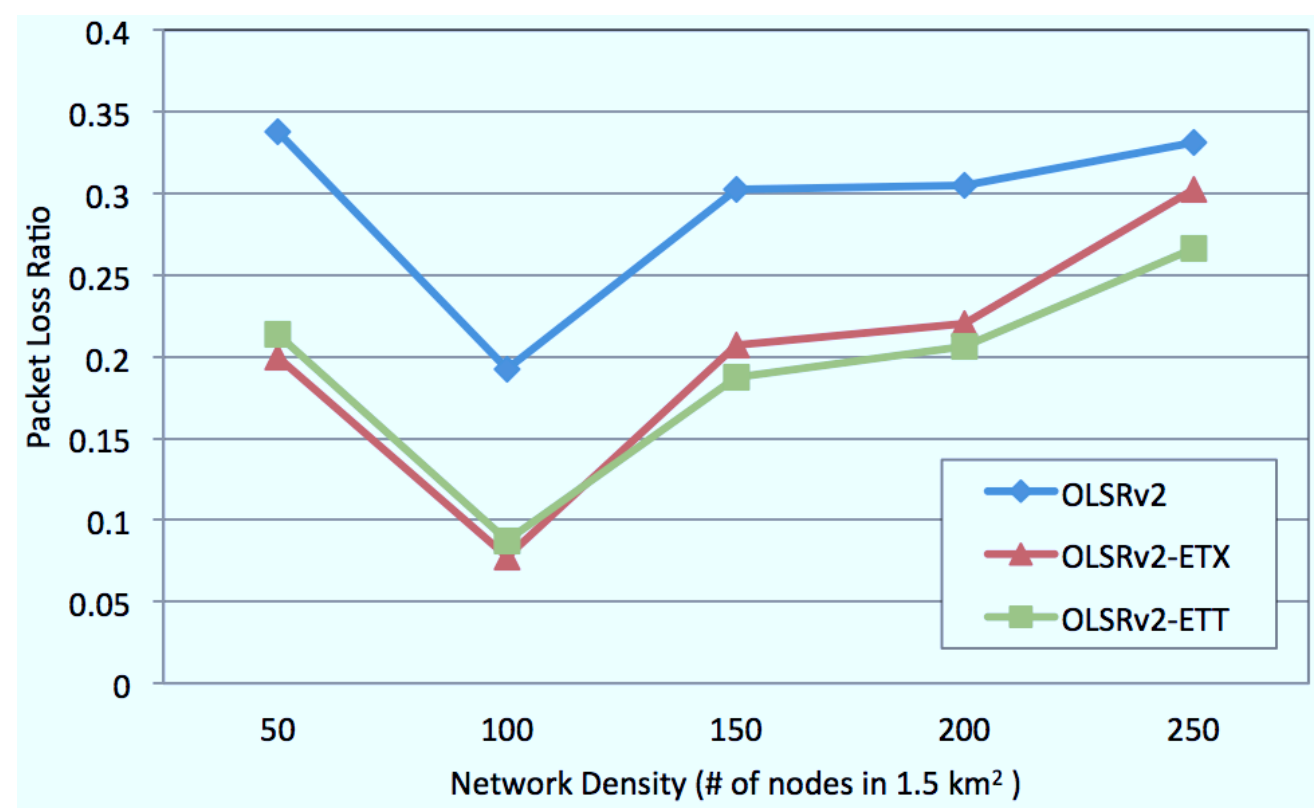

Figure 4.14: Packet loss comparison with various node densities 


\subsection{Summary}

The results in this chapter indicate that the ETT metric outperforms the ETX and hop count metrics under the conditions of varying flow rates and network density. Both ETX and ETT establish longer routes than hop count, as they account for link quality while assigning weights. Although these routes are longer, the quality factor plays a huge role in guaranteeing better performance.

Hop count ignores the quality of links along the path. Hence, it proved to be the least desirable metric. When the network density was increased, ETX and ETT outperformed hop count, as there were more optional routes for them to select quality links from. However, hop count has the least impact of the node density. When the flow rates increased, ETX and ETT avoided congested routes and showed better performance. As expected, ETX which does not consider link capacity, performed worse than ETT. While, hop count established stable routes along congested paths; but experienced increased losses as the flow rate increased. However, in certain scenarios, when packet loss increased, it was occasionally found to perform better in comparison to ETX.

But, when network density was increased it was found to be more stable than ETX and ETT. ETX and ETT outperformed hop count when the network density increased, as there were more optional routes for them to select quality links from.

For a given end-to-end node pair, there are multi-paths between them. Any changing condition will cause small fluctuations in the metric values of the path, resulting in ETX and ETT recalculating/rerouting the traffic. Although this can lead to ETX and ETT always choosing better quality paths, it also increases the instability of routes. In the next chapter, we present the performance of the three metrics under different Internet traffic types. 


\section{Chapter 5}

\section{Whitby Area Scenario}

\subsection{Introduction}

In this chapter, we present a scenario based on the wireless mesh topology of a suburban residential area in New Zealand, and analyze the performance of three common Internet traffic types, VoIP, FTP and HTTP traffic in terms of throughput, end-to-end delay and packet loss ratio, and present findings that are closer to the perspective of what an end user experiences.

\subsection{Simulation Set up}

\subsubsection{Whitby Area Scenario}

VillageNet is a community wireless mesh network which provides high speed local connection and community based connection to the Internet via fiber optic cable, land based wireless or satellite. The community network is self contained, inclusive of its own local ISP, enabling shared high speed data connectivity and internet phone at economical rates [53].

In the Greater Wellington region of New Zealand, the Porirua VillageNets Project has worked with the Porirua Chamber of Commerce to 
carry out market research to identify target businesses and high value subscribers. Their objective is to ensure that network coverage is provided to core subscriber centers as effectively as possible. We used a part of their deployment area, in the Whitby Area, as the basis of our simulations. Figure 5.1 is the Whitby Area deployment by Porirua VillageNets. The network consists of 126 nodes deployed in a $2000 \mathrm{~m} \times 2000 \mathrm{~m}$ suburban residential area, and every node is placed on the roof of each house.

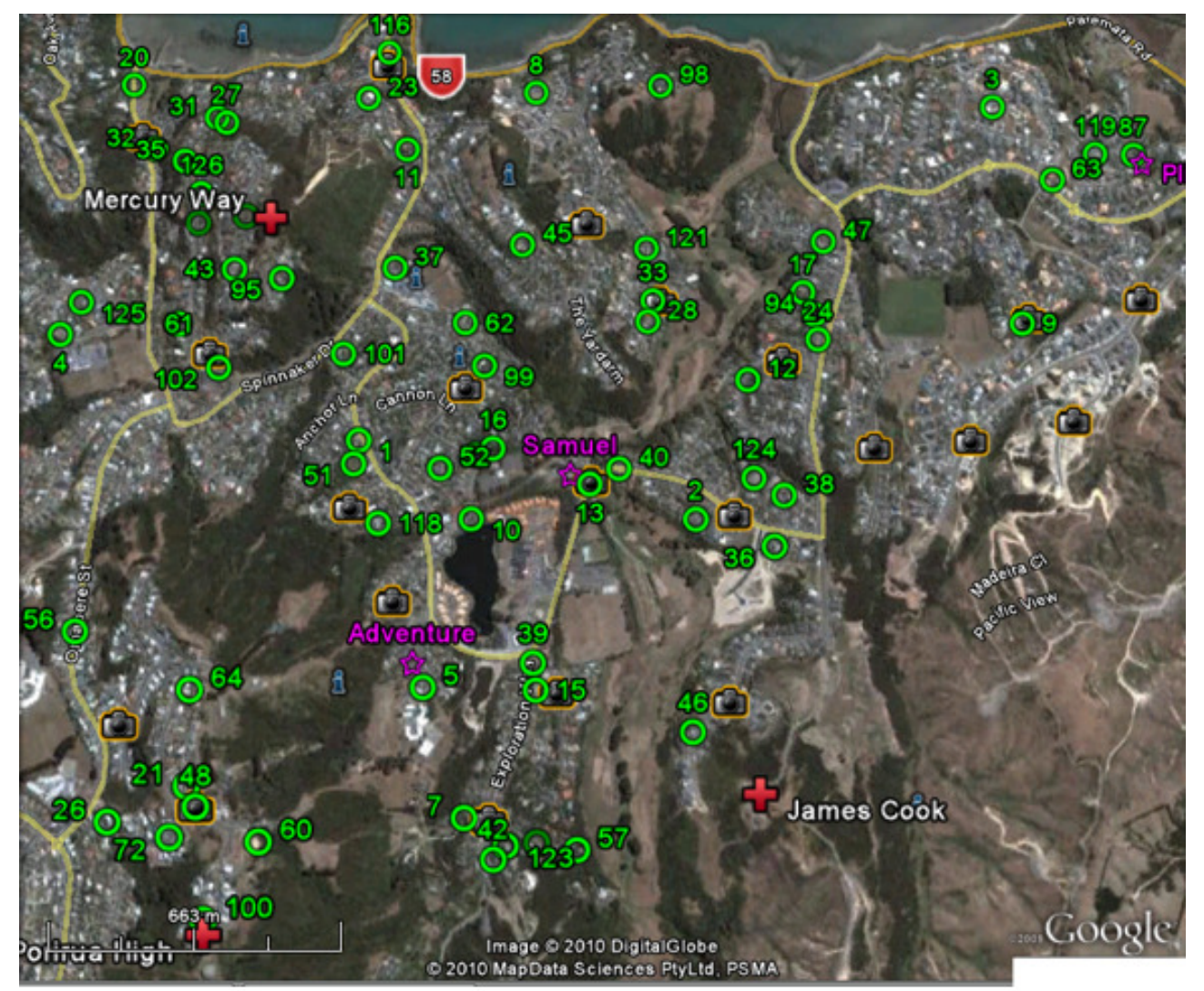

Figure 5.1: Whitby area network topology 


\subsubsection{Simulation Environment}

The simulation, OLSRv2, ETX and ETT parameters of this scenario are the same as in Chapter 4 . The transmitting power of each node was set to $15 \mathrm{~dB}$ and the channel frequency to $2.4 \mathrm{GHz}$. Every node was equipped with one $802.11 \mathrm{~b}$ radio and transmitted data over a single channel. As all the nodes were static, Constant Shadowing and Two Ray path loss models were used in the simulation. The antenna model was omni-directional. The various simulation parameters' values are summarized in Table 5.1.

\begin{tabular}{|l|c|}
\hline Simulation Parameter & Value \\
\hline Simulation Area & $2000 \mathrm{~m}{ }^{*} 2000 \mathrm{~m}$ \\
\hline Number of Nodes & 126 \\
\hline Simulation Time & $300 \mathrm{~s}$ \\
\hline Physical Model & $802.11 \mathrm{~b}$ \\
\hline Channel Frequency & $2.412 \mathrm{GHz}$ \\
\hline Data Rate & $11 \mathrm{mbps}$ \\
\hline Transmission Power & $15 \mathrm{dBm}$ \\
\hline PathLoss Model & Two Ray \\
\hline Shadowing Model & Constant \\
\hline Antenna Model & Omni-directional \\
\hline
\end{tabular}

Table 5.1: Simulation Parameter

We used VoIP, HTTP and FTP traffic types. In every traffic type, we varied the traffic loads in the network from 10 to 50 connections. The reason for configuring the number of flows was to analyze the performance under different traffic loads for audio, bulk file transfer and web streams. For every scenario, more than twenty simulations were performed using different seeds to get the average results. Each simulation was run for 600 seconds. 


\subsection{Simulation Results}

\subsubsection{Voice over Internet Protocol (VoIP) Traffic}

Nowadays VoIP has emerged as a reliable telecommunication technology that is widely used to compete against traditional phone services. QoS provisioning is an essential element for high quality VoIP calls. To improve the quality of VoIP calls, the key QoS parameters are delay, jitter and packet loss ratio. We examined OLSRv2 using the hop count, ETX and ETT metrics with regard to these parameters.

For signaling and control, we used H.323 [54] and Real-time Transport Protocol (RTP) [55]. As VoIP data is small, the UDP flow rate is maintained at $8 \mathrm{~Kb} / \mathrm{s}$, packet size at 32 bytes (RTP + UDP + Payload) and packetisation interval at $20 \mathrm{~ms}$ to closely emulate the G.729 codec [56]. We randomly selected pairs of VoIP communicating nodes from the nodes in the network.

In Figure 5.2, we have plotted the average end-to-end delay experienced by the VoIP packets. It is obvious that the ETX and ETT metrics reduce end-to-end delay when the traffic load is low. When 10 VoIP connections exist, which means twenty nodes are communicating at the same time, the ETX and ETT metrics experience almost 50\% less delay than hop count. When the traffic load increases, the end-to-end delay of all the metrics increase rapidly. From 10 connections to 30 connections, the delay is consistent to hop count, ETX and ETT. When the numbers of connections exceed 40, the hop count metric is able to achieve lower end-to-end delay compared to the ETX metric. However, the ETT metric always provides the lowest end-to-end delay than the other two, even if the traffic load is very high.

When using hop count, as the traffic load increases, the queuing delay increase leading to higher end-to-end delay. Using the hop count metric disregards these intermediate and sudden increases in delays and still tries to establish the shortest path. This will lead to higher packet losses, as shown in Figure 5.4. The ETX and ETT metrics tend to choose a low packet 


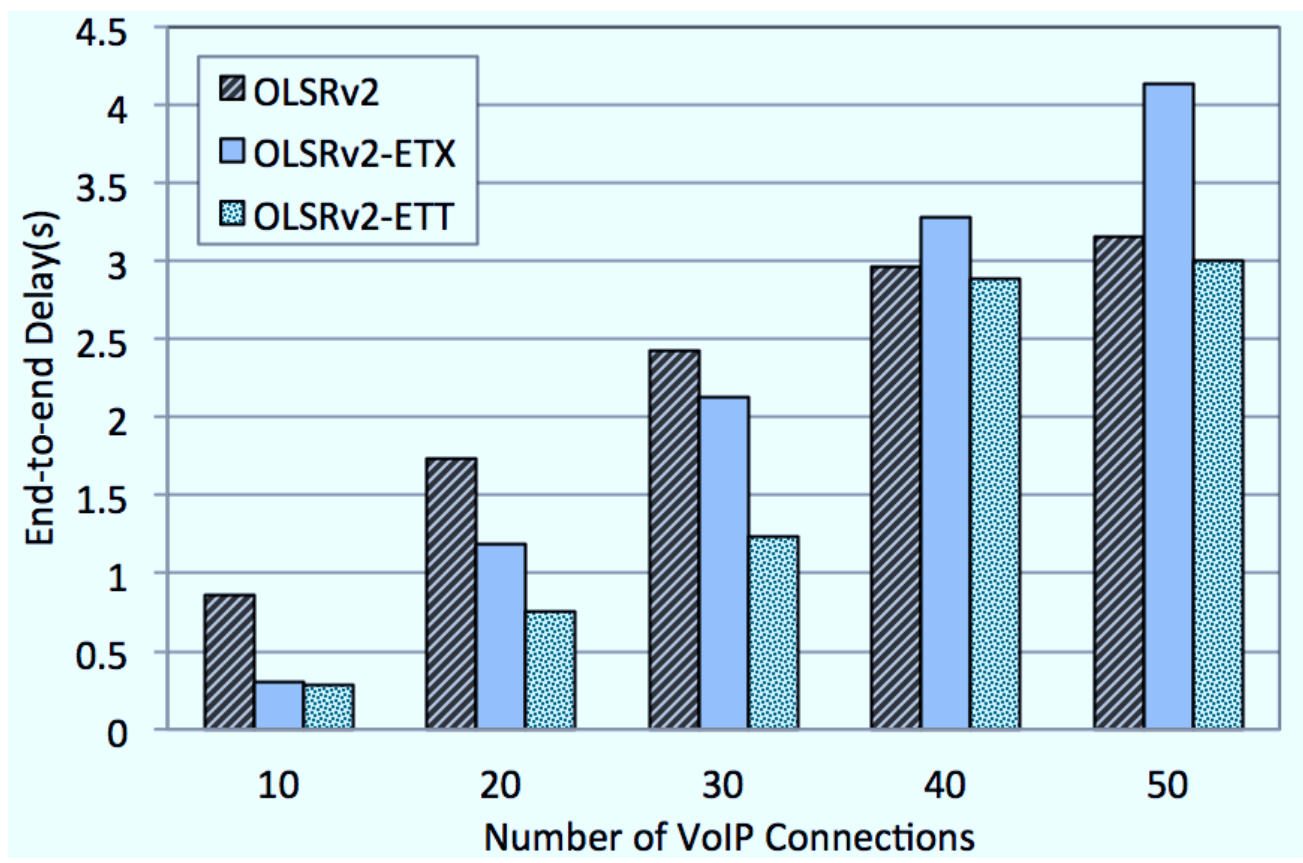

Figure 5.2: Average end-to-end delay

loss path to avoid congestion. In other words, both of them will choose a longer path than hop count. When the traffic load is high enough, ETX and ETT will choose a much longer path to avoid congested areas, resulting in higher end-to-end delay as compared to the hop count metric. On the other hand, when hop count is used, fewer packets reach the destination but those that do have lower delays and thus, the average delay and jitter are lower than those for ETX. When the traffic load is high, ETT also chooses a much longer path than hop count. However, the path ETT chosen has higher link capacity, so end-to-end delay is lower than hop count and ETX.

Figure 5.3 presents the jitter results for VoIP calls. The results are consistent with the end-to-end delay. At low traffic loads, ETX and ETT experience lower jitter compared to hop count. As the traffic load increases, to avoid congestion ETX may change routes, as and when a new route with lower ETX value than existing routes is discovered. This can lead 


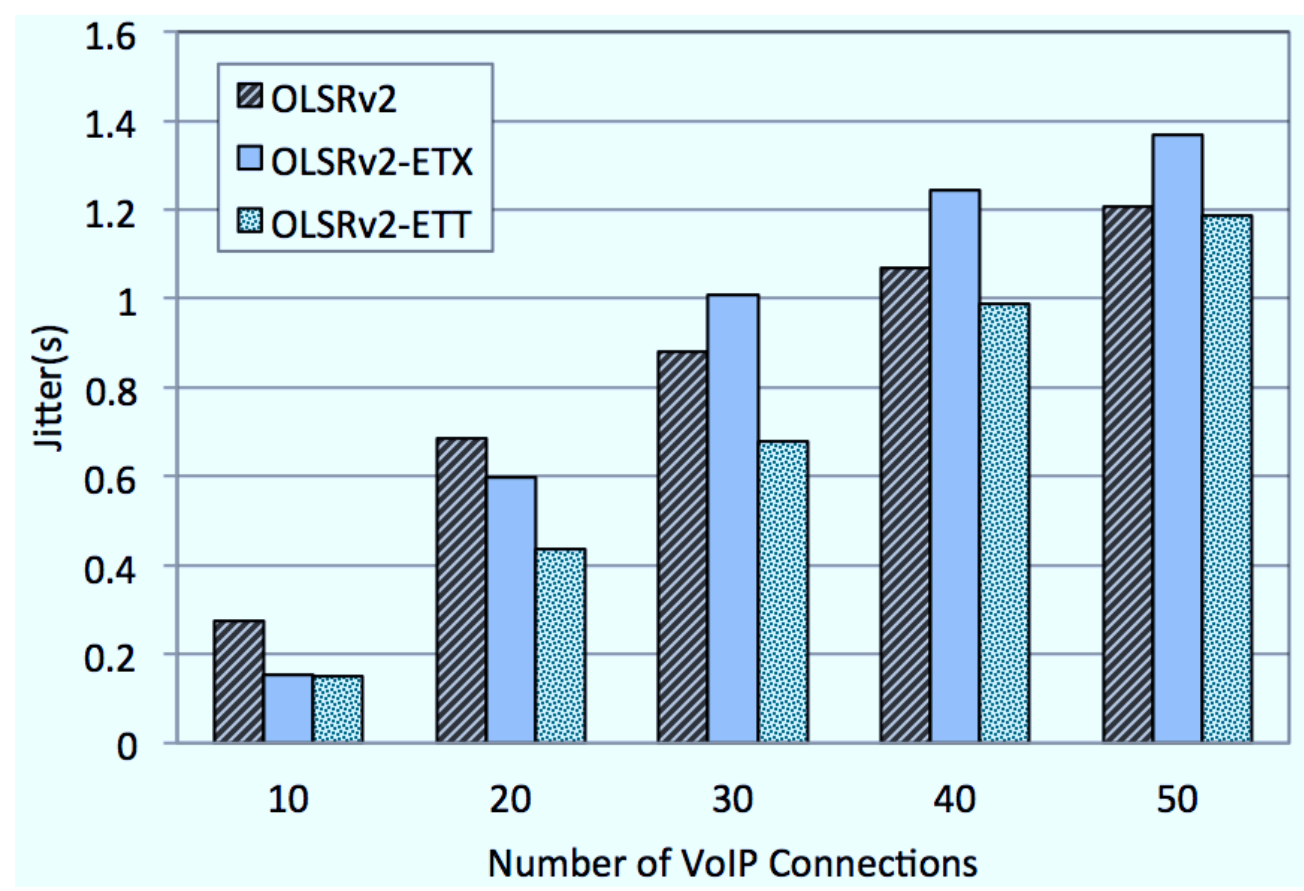

Figure 5.3: Average jitter

to a number of route changes resulting in higher jitter than hop count. The ETT metric takes link bandwidth into account, which provides better quality routes compared to ETT even the traffic load is high.

Figure 5.4 shows the packet loss ratio of three metrics. Irrespective of whether the traffic load is low or high, it is observed that the ETX and ETT metrics, which take packet loss ratio into account, ensure a much lower packet loss than hop count. Hop count is indifferent to packet losses caused by aggravated congestion and interference along the shortest routes, invariably resulting in high packet loss ratio. Although the ETX and ETT metrics choose a route with more hops, it keeps the packet loss probability within an acceptable threshold. Because ETT always assigns lower weights to a route with high capacity links, it shows a slightly lower packet loss ratio than ETX. This is significant for VoIP users, as low loss ratio results in better quality of calls. 


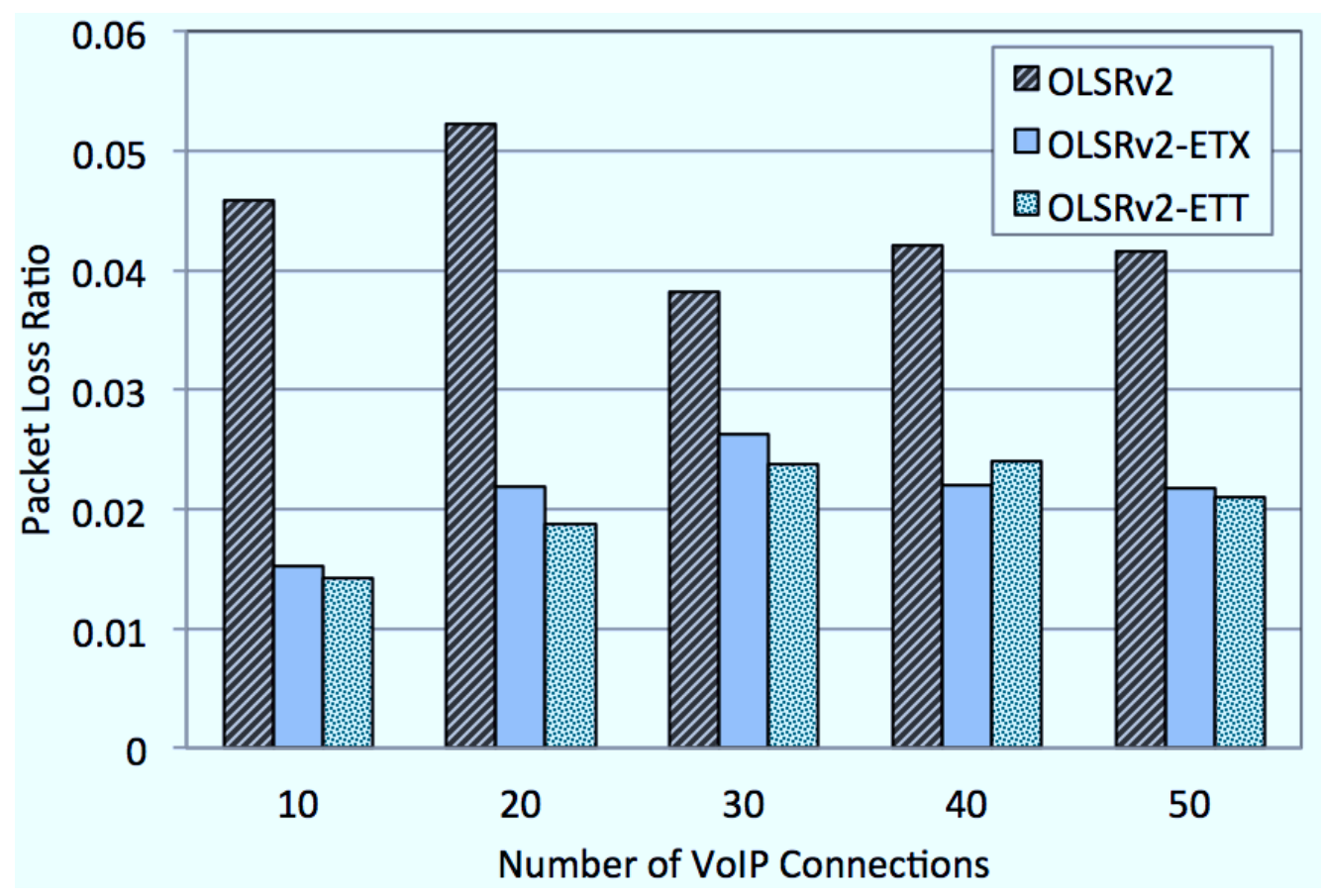

Figure 5.4: Average packet loss ratio

The standard deviation of the packet loss ratio is also shown in Figure 5.5. The lower standard deviation achieved by ETX and ETT shows that the network traffic is distributed across the network, unlike hop count that tends to concentrate traffic along the direct paths between communicating nodes. However, when the traffic load is high, the network becomes congested and route selection based on all approaches experience high performance variations. In general, the standard deviation is lower when the ETX and ETT metrics are used as compared to hop count, since ETX and ETT spread the traffic and avoid the congested routes. The standard deviation of ETT is slightly better than ETT, because ETT takes link capacity into account.

Note that in Figure 5.5, 5.8, 5.10 and 5.12, some standard deviations values are larger than the mean. This is because the path length of individual connections vary from 2 to 15 hops. This causes packet loss, end-to-end 
delay, average page requests time and throughput values to differ by a large margin, especially with more connections and higher traffic loads.

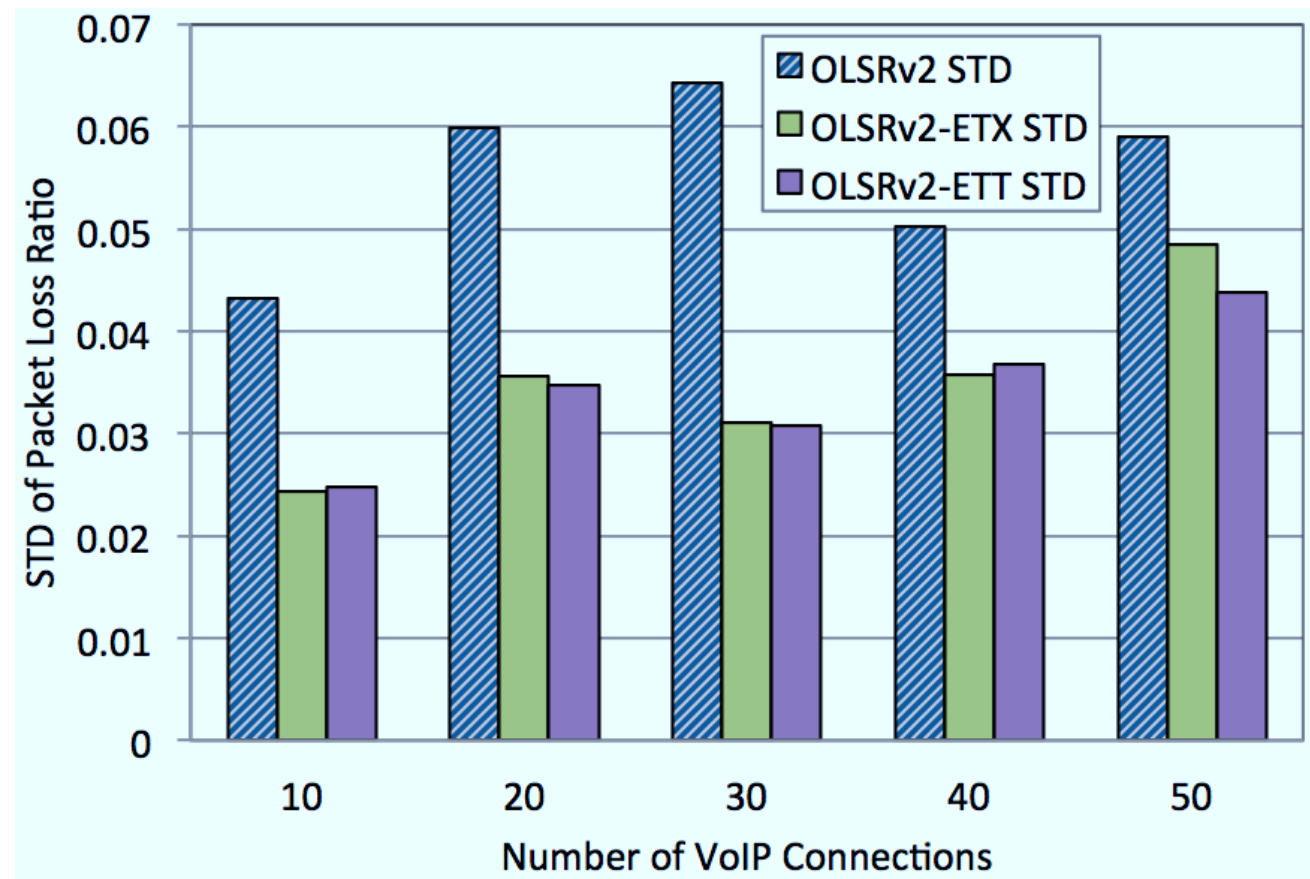

Figure 5.5: Standard deviations of packet loss ratio

Like any routing protocol, OLSRv2 also incurs overheads due to broadcast control messages. In OLSRv2, TC messages cause much larger overheads than Hello messages. Figure 5.6 presents the average number of control messages broadcast by every node. It can be seen that using the ETX and ETT metrics OLSRv2 generates more control messages than using the hop count metric, which will result in significant overheads. The reason is that when using ETX and ETT as its routing metric, OLSRv2 has to select more MPRs to cover links of acceptable link quality values in order to calculate the smallest weighted path. As more MPRs are selected based on the ETX and ETT metrics, more control messages are broadcast across the entire network. 


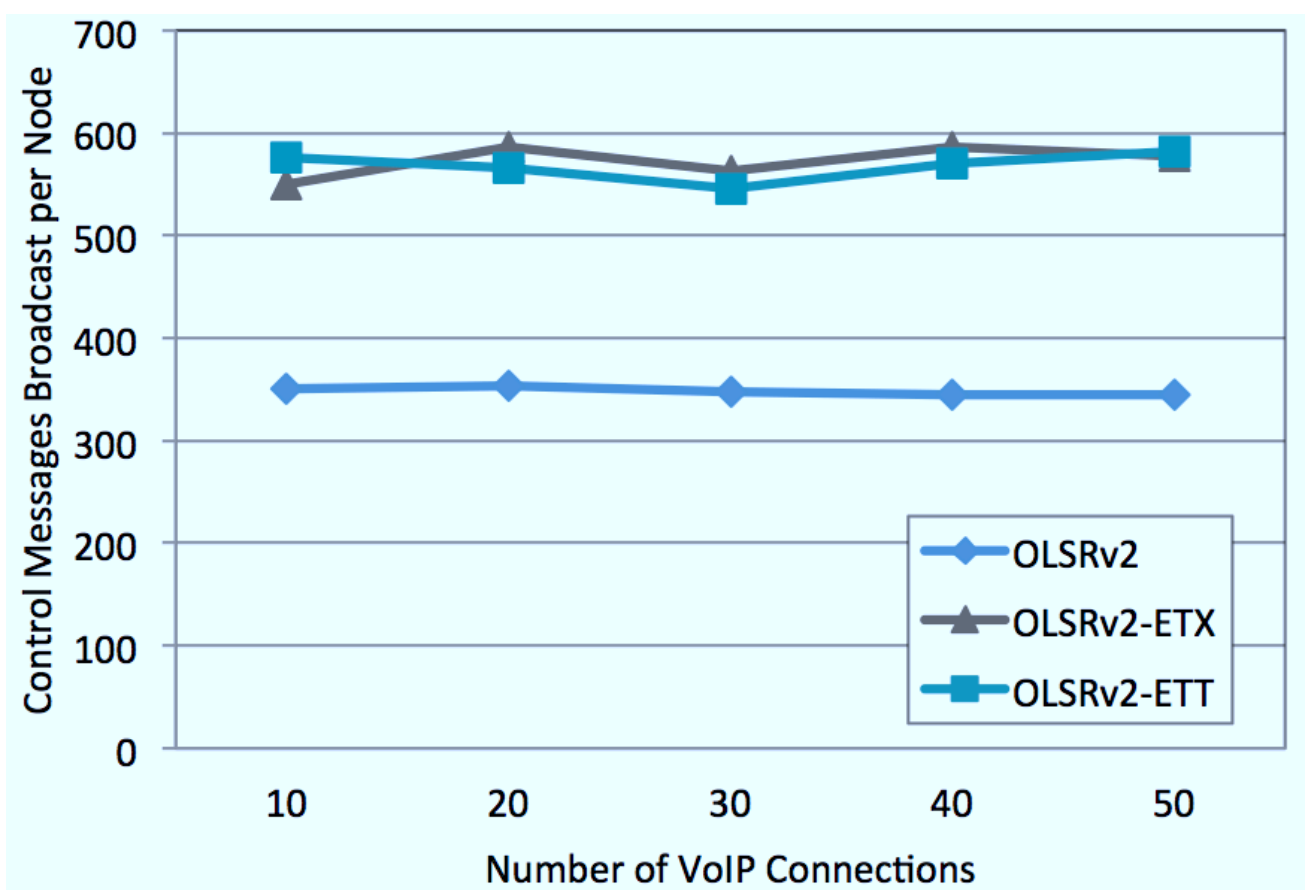

Figure 5.6: Number of control messages broadcast per node

\subsubsection{File Transfer Protocol (FTP) Traffic}

In today's Internet, most traffic is carried by Transmission Control Protocol (TCP), and most of the bytes are carried by long-lived TCP flows [57]. FTP traffic, which is carried by long-live TCP flows, is expected to form a significant portion of the traffic on community wireless networks. It is important to examine the impact of the three metrics on the performance of FTP traffic.

To model FTP traffic, we randomly assigned nodes as FTP servers and clients and set up connections ranging from 10 to 50. Each FTP flow carries 100 file items with an average size of 1460 bytes and lasts for at least two minutes. For each simulation, we calculated the average throughput of all the connections.

Figure 5.7 shows the throughput performance of the two metrics. The results show that the ETX metric gives better throughput than the hop 
count metric by almost $20 \%$, and ETT gives almost 30\% higher throughput than hop count. As expected ETT establishes routes with low loss probability and high link capacity resulting in connections that can support more data transfer than the hop count metric.

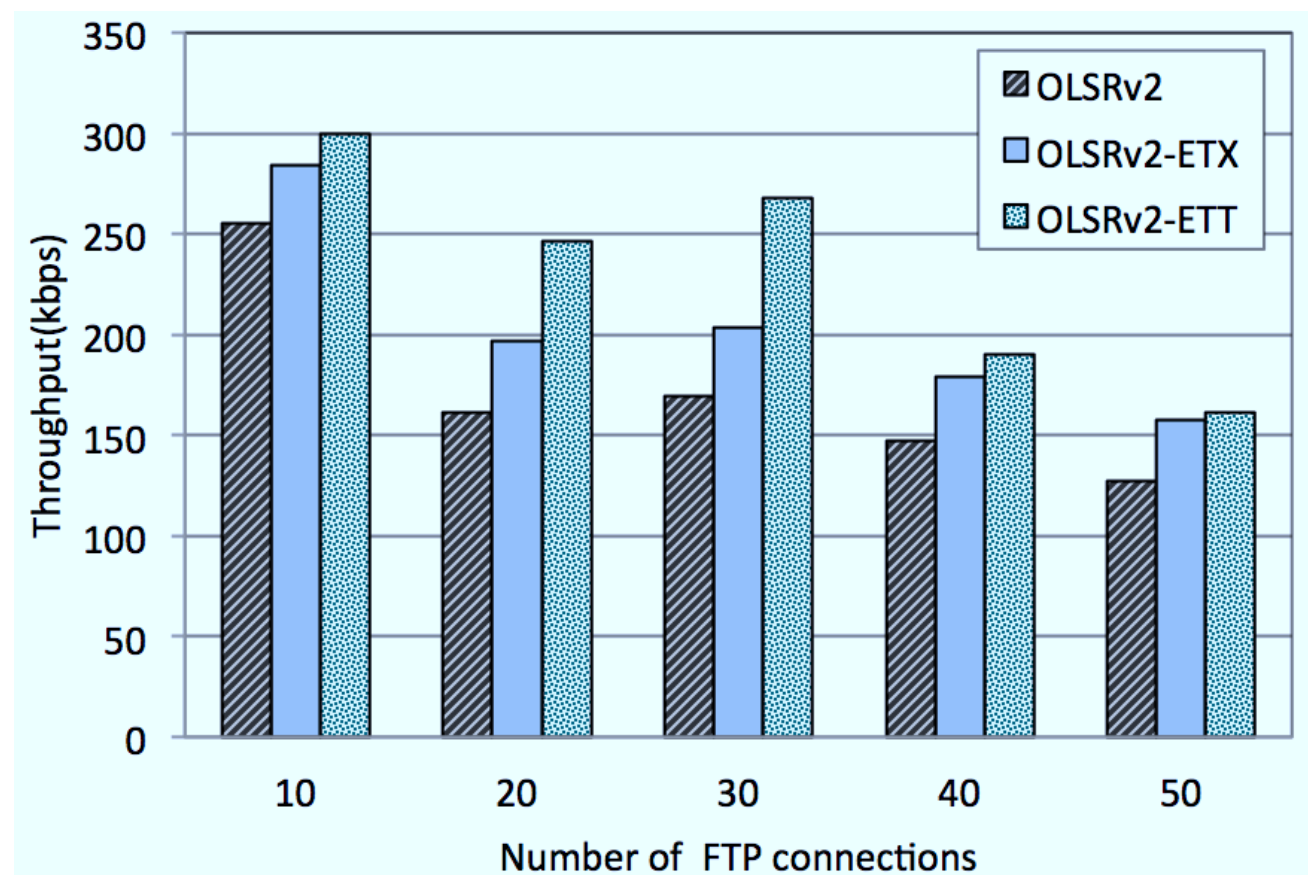

Figure 5.7: Throughput with number of FTP connections

From Figure 5.7, we can see the ETX and ETT metrics provided much better results than the hop count metric when the traffic load was low. Normally longer paths suffer from higher loss probability and lower throughput, but ETX and ETT achieve higher throughput by avoiding these low throughput paths. As the number of connections increased from 10 to 50, the overall throughput of the three metrics reduced. The reason is that links start interfering with one another, and the instability in the ETX and ETT metrics under very high loads. With high traffic loads, the number of poor quality links (lossy or slow) increases. The hop count metric, using Dijkstra's algorithm, still chooses these links to form the shortest path, 
which significantly reduces the throughput. However, when high loads lead to route instability and the overall throughput is considerably lowered, ETT provides comparatively better throughput than hop count and ETT by choosing a high bandwidth path. In Figure 5.8 the lower standard deviation achieved by ETX and ETT still shows that the network traffic is distributed across the network.

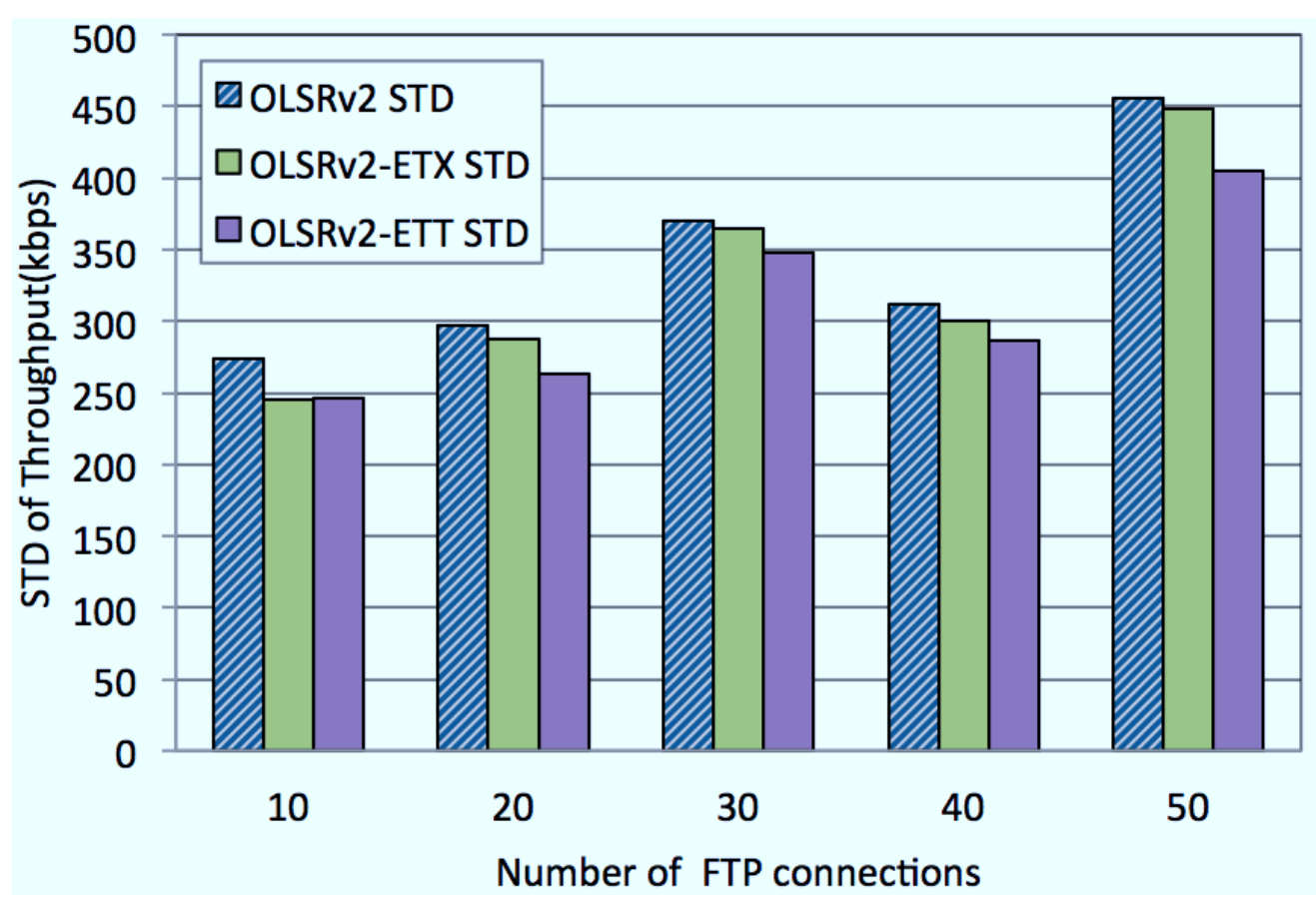

Figure 5.8: Standard deviation of FTP connections' throughputs

\subsubsection{Hypertext Transfer Protocol (HTTP) Traffic}

Web traffic forms a significant portion of today's Internet traffic. To examine the performance of web traffic under various routing metrics is important. In this section to perform web traffic analysis, each client is modeled as a single user running HTTP. It simulates single-TCP connections between web servers and clients. The web traffic was generated using Mah's 
model [58]. HTTP requests are the only data sent from the client to the server and are about 320 bytes, with a median size of 240 bytes. The HTTP server replies, on the other hand, are larger, with median file size around 1.5-2.0 KB. Each user fetched over 40 files from HTTP servers in a session that ran for at least three minutes.

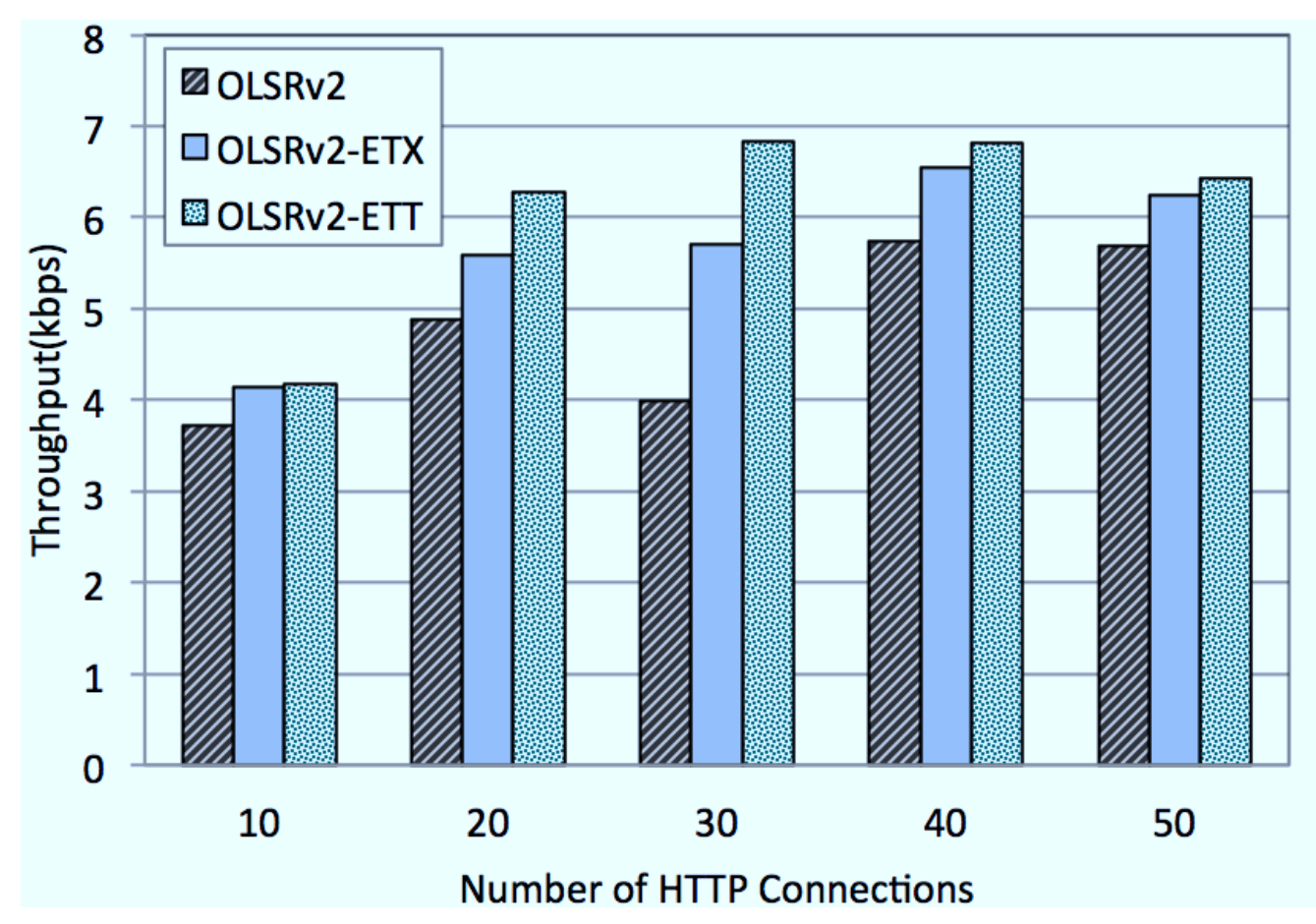

Figure 5.9: Throughput with number of HTTP connections

In Figure 5.9. we plotted the average throughout experienced by each web client which shows that, no matter how much the traffic loads increases, using ETX and ETT can provide higher throughput than using hop count. This is consistent as ETX and ETT are ideal for HTTP traffic as they are high throughput routing metrics. However, contrary to what has been observed about the FTP traffic analyzed earlier, the overall throughput of HTTP does not degrade with the increase in traffic load. One possible reason is that the HTTP servers keep sending web pages to clients, and the TCP connections with HTTP are short-lived compare to the TCP 
connection with FTP, which keeps the throughput high.

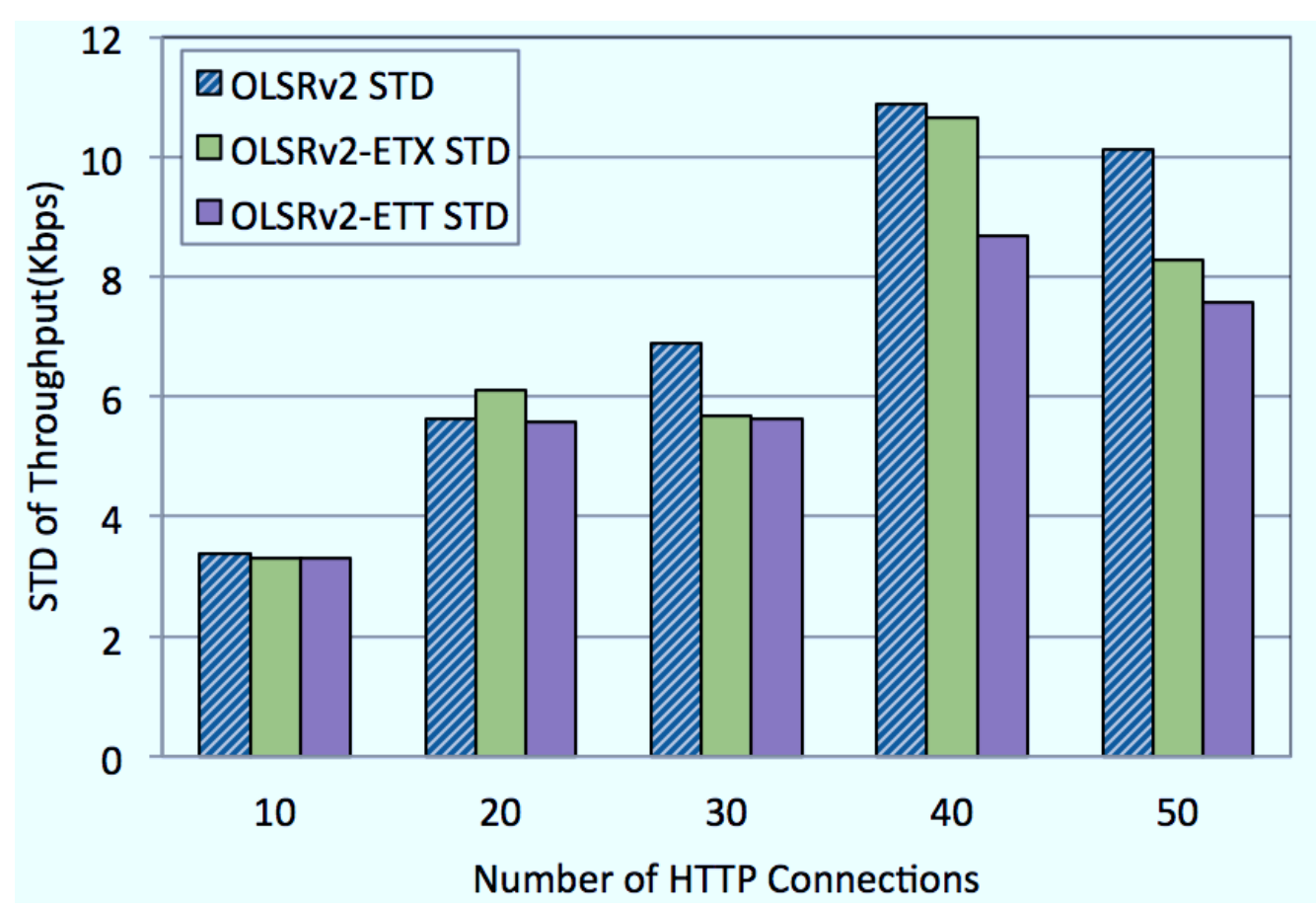

Figure 5.10: Standard deviation of HTTP connections' throughputs

Figure 5.11 presents the average page request time by each web client. The overall request time of hop count, ETX and ETT increase with the increase in traffic load, but ETX and ETT provide at least 50\% lower average request time than hop count. As the request packet is small, ETX and ETT reduce the delay of small transfers by a significant proportion, especially ETT. The request data sent from the client to the server can fit inside a single TCP packet. The standard deviations in Figure 5.12 show that ETX and ETT perform better than hop count, especially when the network is not under extremely high loads. Not only is the HTTP request time lower when ETX and ETT are used, the variations are lesser as shown by the lower standard deviations. This translates to more consistent response times to the end users. ETT shows slightly better throughout than ETT, but it achieves much lower page request time by choosing faster paths. 


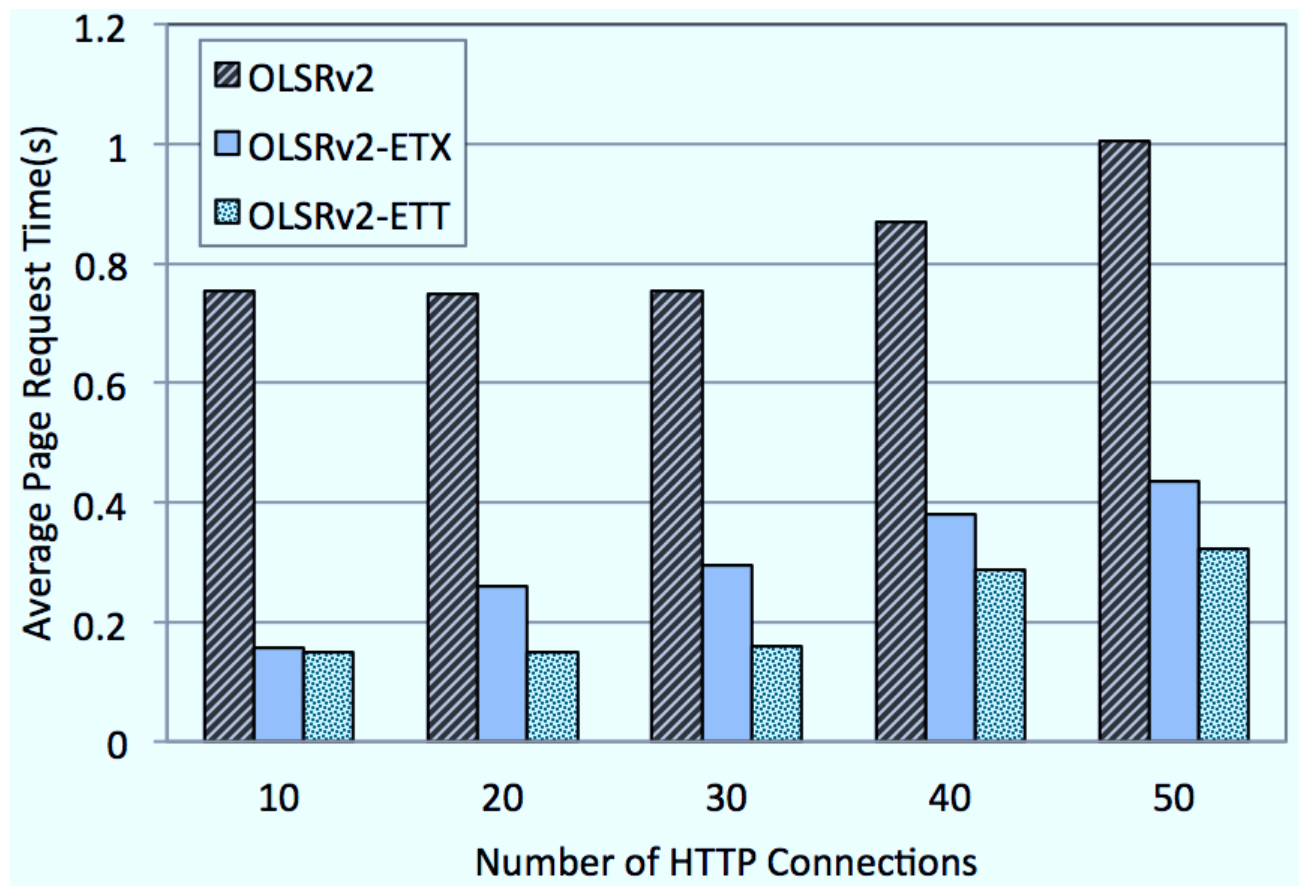

Figure 5.11: Average page request time

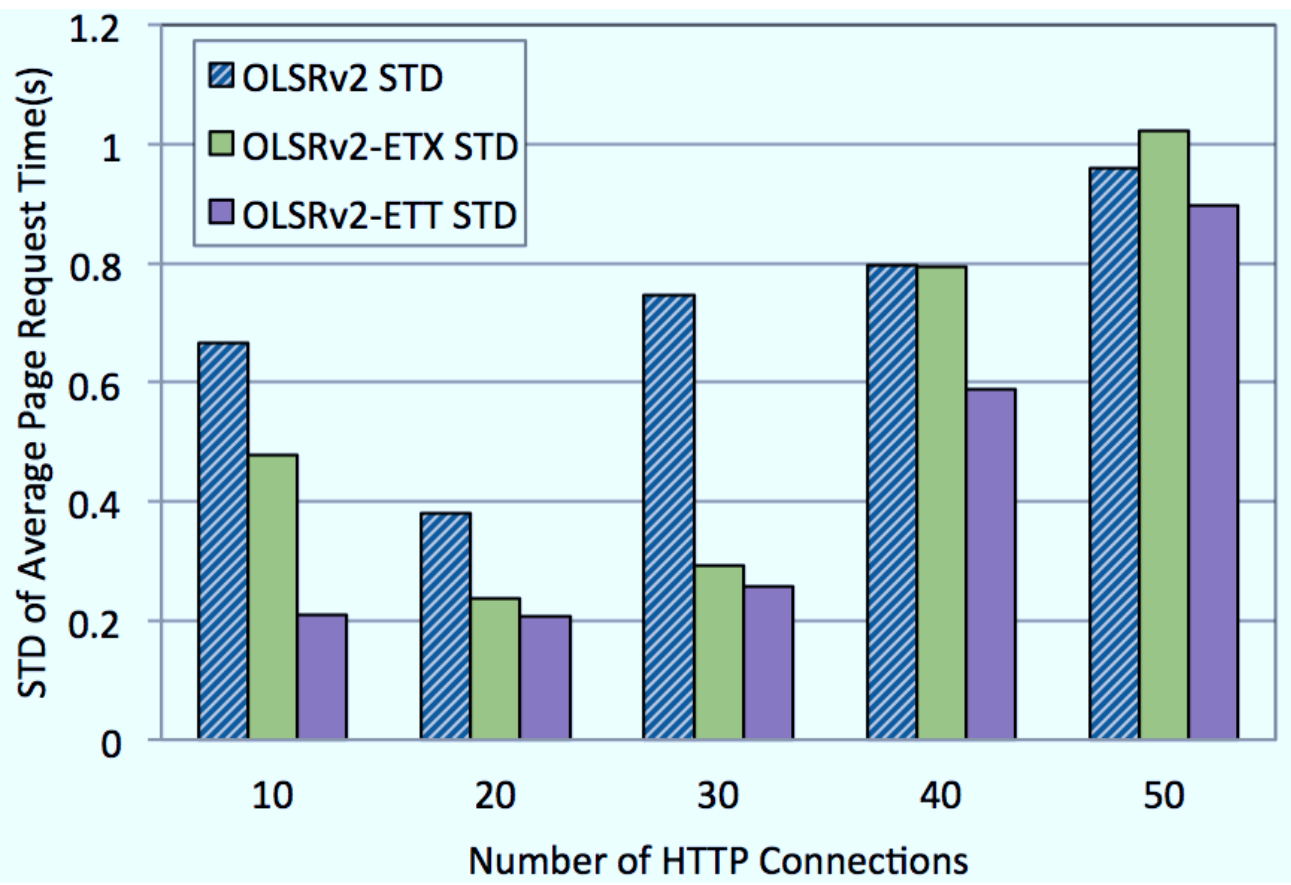

Figure 5.12: Standard deviation of average page request time 


\subsubsection{Summary}

In this chapter, we evaluated the performance of the hop count, ETX and ETT metrics to support three different types of Internet traffic in a network topology based on a real deployment scenario. The simulation results show that using the ETX and ETT metrics in place of the hop count metric improved the overall performance of OLSRv2 protocol, especially in the case of throughput. With increasing traffic loads, performances of all the three metrics reduce rapidly when supporting VoIP traffic. One reason is that VoIP uses UDP as a transport layer protocol which can not provide reliable transmission. Another is high traffic load means more interferences which all the three metrics can not handle. For FTP and HTTP traffic, ETX and ETT guarantee higher throughput because ETX and ETT both consider packet loss probability in both directions of a link, thereby establishing routes with significantly higher throughput than hop count. Because ETT takes link capacity into account, it outperforms the other two metrics in all the scenarios.

On the other hand, when OLSRv2 uses ETX and ETT as its routing metric, it will result in more MPRs and more control messages. The network overheads will increase and significantly reduce the performance of networks. While adopting ETX or ETT, as its routing metric, is a good choice for OLSRv2, we still need to consider the traffic loads, overheads and the traffic profile. We also note that the standard deviation is always lower when ETX and ETT are used, as compared to hop count, under low to moderate traffic conditions for all three types of traffic. When using ETX or ETT as the routing metric, routes that avoid the congested parts or low bandwidth links in the network are selected; under high traffic loads where the network is generally congested throughout, there are far fewer alternative routes to choose from to get any additional benefits from using ETX and ETT. From this perspective, the ETX and ETT metrics are able to provide better traffic equalization than hop count.

The simulation in this chapter still has some limitations that we intend 
to overcome as part of our future work. One such limitation is the use of Hello message in OLSRv2; the Hello messages used by ETX cannot accurately determine the real data loss ratio because the Hello message is quite small compared to the actual data being transmitted. While QualNet provides many propagation models, it is still not entirely possible to model a real deployed network, with the actual buildings' locations, trees, hills or any other reflectors in the line of sight between nodes. Future research should address and implement these shortcomings. 


\section{Chapter 6}

\section{Conclusions}

Since hop count, the most common routing metric, does not meet the requirements for efficient routing in wireless networks, many new routing metrics have been designed to improve the performance of wireless network routing protocols. ETX and ETT are most widely used high throughput metrics in single-channel wireless networks. This thesis has evaluated the performance of the hop count, ETX and ETT metrics with OLSRv2 protocol under different Internet traffic types and network conditions. The simulation results show that ETT outperformed ETX and hop count, and that both ETX and ETT outperformed hop count. Also, the thesis represented how each metric works under different networks conditions and Internet traffic types.

From the results, ETX and ETT selected routes with longer path lengths than hop count, but still provided much better performance. ETX takes packet loss ratio into account, and ETT improves ETX by additionally considering link capacity, which resulted in the paths they selected having higher throughput than hop count. When the flow rates increased, the routes became more congested. However, ETX and ETT avoided these congested routes, resulting in better performance than hop count. When we increased the network density, although ETX and ETT showed better performance, they had more fluctuations than hop count. From the re- 
sults of end-to-end delay and jitter, we could see that the performance of hop count did not change with the node density, as it always selected the shortest path even though the nodes were densely-deployed.

Later, we used a network topology based on a real deployment scenario and increased the network loads to evaluate the three metrics under three Internet traffic types, namely, VoIP, FTP and HTTP traffic. ETT performed better in the case of VoIP traffic, as it improved the performance in terms of end-to- end delay and jitter of the VoIP calls. Both ETX and ETT performed better in terms of throughput than hop count under FTP and HTTP traffic, and ETT showed faster web page request time for the end users.

ETX and ETT take link loss ratio into account, and ETT improves upon ETX by considering link capacity as well. They outperformed the hop count metric, yet they still have shortcomings. In order to cover all the good quality links, OLSRv2 floods more control messages to the entire network, which results in large overheads that significantly reduce the network performance. Also, any small fluctuations in the metric value of paths will cause them to alter routes frequently to avoid low quality links that could result in a higher risk of instability of route selection due to the large and irregular traffic variations. This is one of the reasons why the overall performance is reduced when the network conditions change. The weakness in handling interference also showed they are more suitable for single-radio and single-channel networks. 


\section{Chapter 7}

\section{Future Direction}

The current thesis forms a good baseline for future research, where the performance of new or improved wireless routing metrics in simulation or experimental could be extended and expanded upon the results in previous chapters. Future work can be divided into three main aspects, which are discussed in the following sections.

\subsection{Improvement to the Simulation and Results Analysis}

As we mentioned in Chapter 5, there are some aspects that can be improved in future such as the limitations and accurate modeling of the simulation. More specifically:

- Although we used a part of VillageNets' deployment area as our simulation topology, it is hard to replicate the conditions such as obstacles, weather and reflection in simulations. Implementing other modules or modifying QualNet source code in future can improve the realism of the simulation.

- We used fixed rate for data transfer in our simulations, but Draves 
et.al. in [59] state that the ETX metric performs well in an auto-rate environment, and the auto-rate algorithm can improve the network performance. Awerbuch et. al in [60] proposed a routing algorithm to study the impact of auto-rate selection, which is not based on packet loss and can be used along with the ETX and ETT metrics. These issues can be explored as future work.

- In Chapter 4, we generated CBR traffic which uses UDP as the default transport layer protocol. Due to the traffic shaping characteristics of TCP such as timeouts and window back-offs, there will be more impact on its throughput than that of UDP connections. TCP can amplify the negative impact of poor route selection, which in turn might enhance the performance of ETX and ETT. In future, we wish to use TCP as the transport protocol instead of UDP.

In future, we aim to conduct to theoretical analysis for mathematically representing the improvement brought about by ETX and ETT in comparison to hop count. In addition, statistical modeling techniques/tools should be used to analyze the results to get a deeper understanding.

\subsection{Real Network Experimention}

Using simulations, we can accurately model the behavior of the routing protocols in different network topologies and obtain results in a matter of hours. However, no simulator can comprehensively consider all the conditions in a real wireless network. Even when accurate physical models are used, simulation results cannot closely depict the physical wireless mesh network. Therefore, in the future, we plan to set up a wireless mesh test bed to evaluate these metrics under identical conditions. In addition to providing realistic results, it can help gauge the differences between simulation models and physical experiments. 


\subsection{Multi-Radio and Multi-Channel Environment}

This thesis only considers a single-radio, single- channel environment. However, many research areas on routing metrics are based on multiradio, multi-channel network [42, 61, 62, 63]. Future work on implementing the metrics in a multi-radio and multi-channel wireless mesh network is required. Also other routing metrics likeWCETT and MIC, as mentioned in Chapter 2, should be implemented and compared.

Even though ETX and ETT can both indirectly handle inter-flow interference, hop count, ETX and ETT do not work well with multi-radio and multi-channel networks, as they were not designed to account for interference and channel diversity. However, WCETT and MIC were designed for multi-channel networks in mind and can address performance issues related to interference. Hence, we wish to implement and compare with multi-radio and multi-channel routing metrics such as WCETT and MIC. In addition, we should also look at various means to improve these routing metrics. 


\section{Bibliography}

[1] R. Bruno, M. Conti, and E. Gregori, "Mesh networks: commodity multihop ad hoc networks," IEEE Communications Magazine, vol. 43, no. 3, pp. 123 - 131, Mar 2005.

[2] I. Akyildiz and X. Wang, "A survey on wireless mesh networks," IEEE Communications Magazine, vol. 43, no. 9, pp. S23-S30, Sept 2005.

[3] —, Wireless Mesh Networks. WILEY, 2009, ch. Introduction.

[4] Wireless Ad-hoc and Local Area Networks Research Lab, Homepage: http://www.ece.ncsu.edu/wireless/WMN/.

[5] J. Broch, D. A. Maltz, D. B. Johnson, Y. C. Hu, and J. Jetcheva, "A performance comparison of multi-hop wireless ad doc network routing protocols," in Proceedings of 4th Annual ACM/IEEE ACM International Conference on Mobile Computing and Networking (MobiCom '98), Dallas, Texas, USA, Oct 1998, pp. 85-87.

[6] S. Waharte, B. Ishibashi, R. Boutaba, and D. Meddour, "Performance study of wireless mesh networks routing metrics," in Proceedings of the IEEE/ACS International Conference on Computer Systems and Applications (AICCSA), Doha, Qatar, Mar-Apr 2008.

[7] V. Biradar, Rajashree, and V. Patil, "Classification and comparison of routing techniques in wireless ad hoc networks," in Proceedings of the 
International Symposium on Ad Hoc and Ubiquitous Computing (ISAUHC '06), Mangalore, India, Dec 2006, pp. 7-12.

[8] C. Perkinks, E. Beliding-Royers, and S. Das, "Ad hoc on-demand distance vector (AODV) routing," Internet Engineering Task Force, RFC 3561, Jul 2003.

[9] D. Johnson, Y. Hu, and D.Maltz, “The dynamic source routing protocol (DSR) for mobile ad hoc networks for ipv4," Internet Engineering Task Force, RFC 4782, Feb 2007.

[10] T. Clausen and P. Jacquet, “Optimized link state routing protocol (OLSR)," Internet Engineering Task Force, RFC 3626, Oct 1985.

[11] J. Postel and J. Reynolds, "File transfer protocol (FTP)," Internet Engineering Task Force, RFC 959, Oct 2003.

[12] R. Fielding, J. Gettys, J. Mogul, H. Frystyk, and T. Berners-Lee, "Hypertext transfer protocol - HTTP/1.1," Internet Engineering Task Force, RFC 2068, Jan 1997.

[13] K.-W. Chin, J. Judge, A. Williams, and R. Kermode, "Implementation experience with MANET routing protocols," SIGCOMM Comput. Commun. Rev., vol. 32, no. 5, pp. 49-59, Nov 2002.

[14] T. Goff, N. Abu-Ghazaleh, B. Nael, D. Phatak, and R. Kahvecioglu, "Preemptive routing in ad hoc networks," in Proceedings of the 7th annual international conference on Mobile computing and networking (MobiCom '01), Rome, Italy, Jul 2001, pp. 43-52.

[15] R. Dube, C. Rais, W. Kuang-Yeh, and S. Tripathi, "Signal stabilitybased adaptive routing (SSA) for ad hoc mobile networks," IEEE Personal Communications, vol. 4, no. 1, pp. 36-45, Feb 1997. 
[16] A. Woo, T. Tong, C. Terence, and D. Culler, "Taming the underlying challenges of reliable multihop routing in sensor networks," in Proceedings of the 1st international conference on Embedded networked sensor systems (SenSys '03), Los Angeles, California, USA, Oct 2003, pp. 1427.

[17] A. Zakrzewska, L. Koszalka, and I. Pozniak-Koszalka, "Performance study of routing protocols for wireless mesh networks," in Proceedings of the 19th International Conference on Systems Engineering (ICSENG '08), Las Vegas, USA, Aug 2008, pp. 331-336.

[18] I. Khan and A. Qayyum, "Performance evaluation of aodv and olsr in highly fading vehicular ad hoc network," in Proceedings of the IEEE 13th International Multitopic Conference (INMIC), Islamabad, Pakistan, Dec 2009, pp. 1-5.

[19] X. Zou, B. Ramamurthy, and S. Magliveras, "Routing techniques in wireless ad hoc networks classification and comparison," in Proceeding of 6th World Multiconference on Systemics, Cybernetics and Informatics (SCI 2002), Orlando, Florida, USA, Jul 2002.

[20] D. Rastogi, S. Ganu, Y. Zhang, W. Trappe, and C. Graff, "A comparative study of aodv and olsr on the orbit testbed," in Proceedings of IEEE Military Communications Conference (MILCOM), Orlando, Florida, USA, Oct 2007, pp. 1-7.

[21] S. Waharte, R. Boutaba, Y. Iraqi, and B. Ishibashi, "Routing protocols in wireless mesh networks: Challenges and design considerations," Multimedia Tools Appl., vol. 29, pp. 285 - 303, Jun 2006.

[22] H. Hassanein and A. Zhou, "Routing with load balancing in wireless ad hoc networks," in Proceedings of the 4th ACM international workshop on Modeling, analysis and simulation of wireless and mobile systems (MSWIM '01), Rome, Italy, Jul 2001, pp. 89-96. 
[23] Y. Yang, J. Wang, and R. Kravets, "Designing routing metrics for mesh networks," in Proceedings of the IEEE Workshop on Wireless Mesh Networks (WiMesh), Santa Clara, CA, USA, Sep 2005.

[24] M. Bahr, J. Wang, and X. Jia, Wireless Mesh Networking: Architecture, Protocols and Standers. Auerbach, Dec 2006, no. 4, ch. Routing in Wireless Mesh Networks.

[25] C. Perkins and E. Royer, "Ad-hoc on-demand distance vector routing," in Proceedings of second IEEE Workshop on Mobile Computing Systems and Applications (WMCSA), New Orleans, LA, USA, Feb 1997.

[26] R. Draves, J. Padhye, and B. Zill, "The architecture of the link quality source routing protocol," Microsoft Research, Technical Report MSRTR-2004-57, 2004.

[27] T. Yang, L. Barolli, M. Ikeda, F. Xhafa, and A. Durresi, "Performance analysis of olsr protocol for wireless sensor networks and comparison evaluation with aodv protocol," in Proceeding of International Conference on Network-Based Information Systems (NBIS '09), Indianapolis, USA, Aug 2009, pp. 335-342.

[28] A. Laouiti, P. Muhlethaler, and E. P. A. Najid, "Simulation results of the OLSR routing protocol for wireless network," in Proceedings of the 1st Mediterranean Ad-Hoc Networks workshop (Med-Hoc-Net), Sardegna, Italy, Sept 2002.

[29] T. Clausen, G. Hansen, L. Christensen, and G. Behrmann, “The optimized link state routing protocol, evaluation through experiments and simulation," in Proceedings of IEEE Symposium on Wireless Personal Mobile Communications (WPMC '01), Sept 2001.

[30] H. Sinky and B. Hamdaoui, "Implementation and performance measurement and analysis of olsr protocol," in Proceedings of the 6th ACM 
International Wireless Communications and Mobile Computing Conference (IWCMC '10), New York, USA, Jun 2010, pp. 286-290.

[31] T. Clausen, C. Dearlove, and P. Jacquet, "Optimized link state routing protocol version 2," Internet Engineering Task Force, Internet draft, Feb 2007.

[32] J. Guerin, M. Portmann, and A. Pirzada, "Routing metrics for multiradio wireless mesh networks," in Proceedings of the Telecommunication Networks and Applications Conference (ATNAC), Christchurch, New Zealand, Dec 2007.

[33] L. Ma and M. Denko, "A routing metric for load-balancing in wireless mesh networks," in Proceedings of the 21st International Conference on Advanced Information Networking and Applications Workshops (AINAW '07), Ontario, Canada, May 2007, pp. 409-414.

[34] T. Liu and W. Liao, "Capacity-aware routing in multi-channel multirate wireless mesh networks," in Proceedings of the IEEE International Conference on Communications (ICC '06), Istanbul, Turkey, Jun 2006.

[35] J. L. Sobrinh, "Algebra and algorithms for qos path computation and hop-by-hop routing in the internet," in Proceedings of the Twentieth Annual Joint Conference of the IEEE Computer and Communications Societies (INFOCOM '01), Anchorage, Alaska, USA, Apr 2001.

[36] — " "Network routing with path vector protocols: Theory and applications," in Proceedings of the ACM 2003 Conference on Applications, Technologies, Architectures, and Protocols for Computer Communication(SIGCOMM '03), Karlsruhe, Germany, Aug 2003.

[37] P. Kyasanur and N. Vaidya, "Multi-channelwirelessnetworks: Capacity and protocols," University of Illinois at Urbana-Champaign, Tech. Rep., 2006. 
[38] S.-J. Lee and M. Gerla, "Dynamic load-aware routing in ad hoc networks," in Proceedings of the IEEE International Conference on Communications (ICC '01), Helsinki, Finland, Jun 2001.

[39] C. Perkins and P. Bhagwat, "Highly dynamic destination- sequenced distance-vector routing (DSDV) for mobile computers," in Proceedings of the ACM conference on Communications architectures, protocols and applications (SIGCOMM), London, UK, Aug 1994.

[40] D. Couto, D. S. J., D. Aguayo, J. Bicket, and R. Morris, "A highthroughput path metric for multi-hop wireless routing," in Proceedings of the 9th ACM International Conference on Mobile Computing and Networking (MobiCom), San Diego, CA, USA, Sep 2003, pp. 419-434.

[41] G. Parissidis, M. Karaliopoulos, R. Baumann, T. Spyropoulos, and B. Plattner, Guide to Wireless Mesh Networks. Auerbach, 2009, no. 8, ch. Routing in Wireless Mesh Networks.

[42] R. Draves, J. Padhye, and B. Zill, "Routing in multi-radio, multi-hop wireless mesh networks," in Proceedings of the 10th ACM Annual International Conference on Mobile Computing and Networking (MobiCom '04), Philadelphia, PA, USA, Sep 2004.

[43] S. Keshav, "A control-theoretic approach to flow control," in Proceedings of the ACM 1991 Conference on Applications, Technologies, Architectures, and Protocols for Computer Communication(SIGCOMM '91), Zurich, Switzerland, Sep 1991.

[44] P. M. Esposito, M. Campista, I. M. Moraes, L. Costa, O. Duarte, and M. G. Rubinstein, "Implementing the expected transmission time metric for olsr wireless mesh networks," in Proceedings of the 1st IFIP Wireless Days(WD), Dubai, United Arab Emirates, Nov 2008.

[45] A. Subramanian, M. Buddhikot, and S. Miller, "Interference aware routing in multi-radio wireless mesh networks," in Proceedings of the 
2nd IEEE Workshop on Wireless Mesh Networks (WiMesh), Reston, VA, USA, Sep 2006.

[46] Y. Yang, J. Wang, and R. Kravets, "Interference-aware load balancing for multihop wireless networks," University of Illinois at Urbana Champaign, Tech. Rep. UIUCDCS-R-2005-2526, Mar 2005, http: //www.ideals.uiuc.edu/handle/2142/10974.

[47] H. Liu, X. Z. W. Huang, and X. Wang, "A comprehensive comparison of routing metrics for wireless mesh networks," in Proceedings of the IEEE International Conference on Networking, Sensing and Control (ICNSC), Sanya, China, Apr 2008.

[48] T. Clausen, C. Dearlove, J. Dean, and C. Adjih, “Generalized mobile ad hoc network (MANET) packet/message format," Internet Engineering Task Force, RFC 5444, Feb 2009.

[49] Information and Communication Networks Laboratory, http:/ / www2.net.ie.niigata-u.ac.jp/nuOLSRv2/.

[50] Y. Ge, T. Kunz, and L. Lamont, "Quality of service routing in ad-hoc networks using olsr," in Proceedings of the 36th Annual Hawaii International Conference on System Sciences (HICSS-36), Big Island, Hawaii, USA, Jan 2003.

[51] C. Dearlove, T. Clausen, and P. Jacquet, "Link metrics for OLSRv2 draft-dearlove-olsrv2-metrics-05," Internet Engineering Task Force, Internet-Draft 05, Jun 2010.

[52] Scalable Networks Technologies, QualNet Network Simulator, Homepage: http://www.scalable-networks.com/.

[53] VillageNet Community Wireless Networks, http://www.villagenet.org.nz/. 
[54] ITU-T Recommendation H.323 version 4, "Packet-based multimedia communications systems," ITU, Nov 2000.

[55] H. Schulzrinne, S. Casner, R. Frederick, and V. Jacobson, "RTP: A transport protocol for real-time applications," Internet Engineering Task Force, RFC 3550, Jul 2003.

[56] J. Balam and J. Gibson, "Multiple descriptions and path diversity for voice communications over wireless mesh networks," IEEE Transactions on Multimedia (TMM '07), vol. 9, no. 5, pp. 1073-1088, Aug 2007.

[57] P. Huang and J. Heidemann, "Capturing tcp burstiness for lightweight simulation," in Proceedings of the SCS Multicoference on Distributed Simulation, Phoenix, Arizona, USA, Jan 2001.

[58] B. Mah, "An empirical model of http network traffic," in Proceedings of Sixteenth Annual Joint Conference of the IEEE Computer and Communications Societies (INFOCOM '97), Kobe, Japan, Apr 1997.

[59] R. Draves, J. Padhye, and B. Zill, "Comparison of routing metrics for static multi-hop wireless networks," in Proceedings of the 2004 conference on Applications, technologies, architectures, and protocols for computer communications (SIGCOMM '04), New York, USA, Aug-Sept 2004, pp. 133-144.

[60] Y. Yang, J. Wang, and R. Kravets, "Throughput route selection in multi-rate ad hoc wireless networks," Johns Hopkins CS Dept, Tech. Rep. 02, Mar 2003.

[61] S. Ghannay, S. Gammar, F. Filali, and F. Kamoun, “Multi-radio multichannel routing metrics in ieee 802.11 s-based wireless mesh networks - and the winner is..." in Proceedings of the first International Conference on Communications and Networking, 2009 (ComNet '09), Hammamet, Tunisia, Nov 2009, pp. 1-8. 
[62] D. Z. W. Zhou and D. Qiao, "Comparative study of routing metrics for multi-radio multi-channel wireless networks," in Proceedings of the 2006 IEEE Wireless Communications and Networking Conference (WCNC '06), Las Vegas, USA, Apr 2006, pp. 270-275.

[63] S. Kim, O. Lee, S. Choi, and S.-J. Lee, "Mac-aware routing metric for 802.11 wireless mesh networks," in 2009 IEEE 20th International Symposium on Personal, Indoor and Mobile Radio Communications (PIMRC '09), Tokyo, Japan, Sept 2009, pp. 47-51. 